\title{
Observing gravitational-wave transient GW150914 with minimal assumptions
}

\author{
B. P. Abbott et al. \\ (LIGO Scientific Collaboration and Virgo Collaboration) \\ (Received 25 February 2016; published 7 June 2016; corrected 20 September 2016)
}

\begin{abstract}
The gravitational-wave signal GW150914 was first identified on September 14, 2015, by searches for short-duration gravitational-wave transients. These searches identify time-correlated transients in multiple detectors with minimal assumptions about the signal morphology, allowing them to be sensitive to gravitational waves emitted by a wide range of sources including binary black hole mergers. Over the observational period from September 12 to October 20, 2015, these transient searches were sensitive to binary black hole mergers similar to GW150914 to an average distance of 600 Mpc. In this paper, we describe the analyses that first detected GW150914 as well as the parameter estimation and waveform reconstruction techniques that initially identified GW150914 as the merger of two black holes. We find that the reconstructed waveform is consistent with the signal from a binary black hole merger with a chirp mass of $\sim 30 \mathrm{M}_{\odot}$ and a total mass before merger of $\sim 70 \mathrm{M}_{\odot}$ in the detector frame.
\end{abstract}

DOI: 10.1103/PhysRevD.93.122004

\section{INTRODUCTION}

The newly upgraded Advanced LIGO observatories $[1,2]$, with sites near Hanford, Washington (H1), and Livingston, Louisiana (L1), host the most sensitive gravitational-wave detectors ever built. The observatories use kilometer-scale Michelson interferometers that are designed to detect small, traveling perturbations in space-time predicted by Einstein $[3,4]$, and thought to radiate from a variety of astrophysical processes. Advanced LIGO recently completed its first observing period, from September 2015 to January 2016. Advanced LIGO is among a generation of planned instruments that includes GEO 600, Advanced Virgo, and KAGRA; the capabilities of this global gravitational-wave network should quickly grow over the next few years [5-8].

An important class of sources for gravitational-wave detectors are short duration transients, known collectively as gravitational-wave bursts [9]. To search broadly for a wide range of astrophysical phenomena, we employ unmodeled searches for gravitational-wave bursts of durations $\sim 10^{-3}-10 \mathrm{~s}$, with minimal assumptions about the expected signal waveform. Bursts may originate from a range of astrophysical sources, including core-collapse supernovae of massive stars [10] and cosmic string cusps [11]. An important source of gravitational-wave transients are the mergers of binary black holes (BBH) [12-14]. Burst searches in data from the initial generation of interferometer detectors were sensitive to distant $\mathrm{BBH}$ signals from mergers with total masses in the range $\sim 20-400 \mathrm{M}_{\odot}$ $[15,16]$. Since burst methods do not require precise

*Full author list given at the end of the article. waveform models, the unmodeled search space may include $\mathrm{BBH}$ mergers with misaligned spins, large mass ratios, or eccentric orbits. A number of all-sky, all-time burst searches have been performed on data from initial LIGO and Virgo [17-19]. Recent work has focussed on improving detection confidence in unmodeled searches, and the last year has seen several improvements in the ability to distinguish astrophysical signals from noise transients [20-24]. As a result, burst searches are now able to make high confidence detections across a wide parameter space.

On September 14, 2015, an online burst search [25] reported a transient that clearly stood above the expected background from detector noise [26]. The alert came only 3 min after the event's time stamp of 09:50:45UTC. A second online burst search independently identified the event with a latency of a few hours, providing a rapid confirmation of the signal [23]. The initial waveform reconstruction showed a frequency evolution that rises in time, suggesting binary coalescence as the likely progenitor, and a best fit model provided a chirp mass around $28 M_{\odot}$, indicating the presence of a $\mathrm{BBH}$ signal. Within days of the event, many follow-up investigations began, including detailed checks of the observatory state to check for any possible anomalies [27]. Two days after the signal was found, a notice with the estimated source position was sent to a consortium of astronomers to search for possible counterparts [28]. Investigations continued over the next several months to validate the observation, estimate its statistical significance, and characterize the astrophysical source $[29,30]$.

In this article, we present details of the burst searches that made the first detection of the gravitational-wave transient, 
GW150914, announced in [26]. We describe results reported in this announcement that are based on the coherent Waveburst algorithm, along with those obtained by two other analyses using omicron-LALInference-Bursts and BayesWave [23,25,31]. In Sec. II, we present a brief overview of the quality of the acquired data and detector performance, before moving on, in Sec. III, to present the three analyses employed. Using each pipeline, we assess the statistical significance of the event. Section IV characterizes each search sensitivity using simulated signals from BBH mergers. In Sec. V, we demonstrate how a range of source properties may be estimated using these same tools-including sky position and masses of the black holes. The reconstructed signal waveform is directly compared to results from numerical relativity (NR) simulations, giving further evidence that this signal is consistent with expectations from general relativity. Finally, the paper concludes with a discussion about the implications of this work.

\section{DATA QUALITY AND BACKGROUND ESTIMATION}

We identify 39 calendar days of Advanced LIGO data, from September 12 to October 20, 2015, as a data set to measure the sensitivity of the searches and the impact of background noise events, known as glitches.

As in previous LIGO, Virgo and GEO transient searches [17-19], a range of monitors tracking environmental noise and the state of the instruments are used to discard periods of poor quality data. Numerous studies have been performed to identify efficient veto criteria to remove nonGaussian noise features, while having the smallest possible impact on detector live time [27].

However, it is not possible to remove all noise glitches based on monitors. This leaves a background residual that has to be estimated from the data. To calculate the background rate of noise events arising from glitches occurring simultaneously at the two LIGO sites by chance [17-19], the analyses are repeated on $\mathcal{O}\left(10^{6}\right)$ independent timeshifted data sets. Those data sets are generated by translating the time of data in one interferometer by a delay of some integer number of seconds, much larger than the maximum $\mathrm{GW}$ travel time $\simeq 10 \mathrm{~ms}$ between the Livingston and Hanford facilities. By considering the whole coincident live time resulting from each artificial time shift, we obtain thousands of years of effective background based on the available data. With this approach, we estimate a false alarm rate (FAR) expected from background for each pipeline.

The "time-shift" method is effective to estimate the background due to uncorrelated noise sources at the two LIGO sites. For the time immediately around GW150914, we also examined potential sources of correlated noise between the detectors, and concluded that all possible sources were too weak to have produced the observed signal [27].

\section{SEARCHES FOR GRAVITATIONAL-WAVE BURSTS}

Strain data are searched by gravitational-wave burst search algorithms without assuming any particular signal morphology, origin, direction or time. Burst searches are performed in two operational modes: online and off-line.

Online, low-latency searches provide alerts within minutes of a GW signal passing the detectors to facilitate follow-up analyses such as searching for electromagnetic counterparts. In the days and weeks following the data collection, burst analyses are refined using updated information on the data quality and detector calibration to perform off-line searches. These off-line searches provide improved detection confidence estimates for GW candidates, measure search sensitivity, and add to waveform reconstruction and astrophysical interpretation. For shortduration, narrowband signals, coherent burst searches have sensitivities approaching those of optimal matched filters [16,32].

In the following subsections, we describe the burst analysis of GW150914. This includes two independent end-to-end pipelines, coherent Waveburst (cWB) and omicron-LALInference-Bursts (oLIB), and BayesWave, which performed a follow-up analysis at trigger times identified by cWB. These three algorithms employ different strategies (and implementations) to search for unmodeled GW transients; hence, they could perform quite differently for specific classes of GW signals. Given the very broad character of burst signals, the use of multiple search algorithms is then beneficial, both to validate results and to improve coverage of the wide signal parameter space.

A summary of the results from cWB has been presented in [26]. Here, we provide more details regarding the cWB search pertaining the discovery of GW150914 and present its results with respect to the other burst searches. In this paper, we focus our characterizations of our pipelines on $\mathrm{BBH}$ sources only.

\section{A. Coherent WaveBurst}

The cWB algorithm has been used to perform all-sky searches for gravitational-wave transients in LIGO, Virgo and GEO data since 2004. The most recent cWB results from the initial detectors are $[17,19,33]$. The cWB algorithm has since been upgraded to conduct transient searches with the advanced detectors [24]. The cWB pipeline was used in the low-latency transient search that initially detected GW150914, reporting the event 3 min after the data were collected. This search aims at rapid alerts for the LIGO/Virgo electromagnetic follow-up program [28] and provides a first estimation of the event parameters and sky location. A slightly different configuration of the same 
pipeline was used in the off-line search to measure the statistical significance of the GW150914 event, which was reported in [26]. The low-latency search was performed in the frequency range of $16-2048 \mathrm{~Hz}$, while the off-line search covered the band of the best detector sensitivity between 16 and $1024 \mathrm{~Hz}$.

\section{1. $c W B$ pipeline overview}

The cWB pipeline searches for a broad range of gravitational-wave transients in the LIGO frequency band without prior knowledge of the signal waveforms [25]. The pipeline identifies coincident events in data from the two LIGO detectors and reconstructs the gravitational-wave signal associated with these events using a likelihood analysis.

First, the data are whitened and converted to the timefrequency domain using the Wilson-Daubechies-Meyer wavelet transform [34]. Data from both detectors are then combined to obtain a time-frequency power map. A transient event is identified as a cluster of time-frequency data samples with power above the baseline detector noise. To obtain a good time-frequency coverage for a broad range of signal morphologies, the analysis is repeated with seven frequency resolutions $\Delta f$ ranging from 1 to $64 \mathrm{~Hz}$ in steps of powers of 2 , corresponding to time resolutions $\Delta t=1 /(2 \Delta f)$ from 500 to $7.8 \mathrm{~ms}$. The clusters at different resolutions overlapping in time and frequency are combined into a trigger that provides a multiresolution representation of the excess power event recorded by the detectors.

The data associated with each trigger are analyzed coherently [24] to estimate the signal waveforms, the wave polarization, and the source sky location. The signal waveforms in both detectors are reconstructed with the constrained likelihood method [35]. The constraint used in this analysis is model independent and requires the reconstructed waveforms to be similar in both detectors, as expected from the close alignment of the $\mathrm{H} 1$ and $\mathrm{L} 1$ detector arms.

The waveforms are reconstructed over a uniform grid of sky locations with $0.4^{\circ} \times 0.4^{\circ}$ resolution. We select the best fit waveforms that correspond to the maximum of the likelihood statistic $L=c_{c} E_{s}$, where $E_{s}$ is the total energy of the reconstructed waveforms ${ }^{1}$ and $c_{c}$ measures the similarity of the waveforms in the two detectors. The coefficient $c_{c}$ is defined as $c_{c}=E_{c} /\left(E_{c}+E_{n}\right)$, where $E_{c}$ is the normalized coherent energy and $E_{n}$ is the normalized energy of the residual noise after the reconstructed signal is subtracted from the data. The coherent energy $E_{c}$ is proportional to the cross-correlation between the reconstructed signal waveforms in $\mathrm{H} 1$ and $\mathrm{L} 1$ detectors. Typically, gravitational-wave signals are coherent and have small residual energy, i.e., $E_{c} \gg E_{n}$ and therefore $c_{c} \sim 1$. On the other hand, spurious noise events (glitches) are often not coherent, and have large residual energy because the

\footnotetext{
${ }^{1} \sqrt{E_{s}}$ is the network signal-to-noise ratio [24].
}

reconstructed waveforms do not fit the data well, i.e., $E_{c} \ll$ $E_{n}$ and therefore $c_{c} \ll 1$. The ranking statistic is defined as $\eta_{c}=\left(2 c_{c} E_{c}\right)^{1 / 2}$. By construction, it favors gravitationalwave signals correlated in both detectors and suppresses uncorrelated glitches.

\section{Classification of $c W B$ events}

Events produced by the cWB pipeline with $c_{c}>0.7$ are selected and divided into three search classes $C 1, C 2$, and $C 3$ according to their time-frequency morphology. The purpose of this event classification is to account for the non-Gaussian noise that occurs nonuniformly across the parameter space searched by the pipeline.

The classes are determined by three algorithmic tests and additional selection cuts. The first algorithmic test addresses a specific type of noise transient referred to as "blip glitches" [27]. During the run, both detectors experienced noise transients of unknown origin consisting of a few cycles around $100 \mathrm{~Hz}$. These blip glitches have a very characteristic time-symmetric waveform with no clear frequency evolution. Previous work has shown that down-weighting signals with simple time-frequency structure can enhance pipeline performance [21]. To implement this here, we apply a test that uses waveform properties to identify, in the time domain, blip glitches occurring at both detectors. The second algorithmic test identifies glitches due to nonstationary narrow-band features, such as power and mechanical resonance lines. This test selects candidates which have most of their energy (greater than 80\%) localized in a frequency bandwidth less than $5 \mathrm{~Hz}$. A cWB event is placed in the search class $C 1$, if it passes either of the aforementioned tests. In addition, due to the elevated nonstationary noise around and below the Advanced LIGO mechanical resonances at $41 \mathrm{~Hz}$, events with central frequency lower than $48 \mathrm{~Hz}$ were also placed in the $C 1$ class.

The third algorithmic test is used to identify events with a frequency increasing with time. The reconstructed timefrequency patterns can be characterized by an ad hoc parameter $\mathcal{M}$ following Eq. (1) in Sec. V D. For coalescing binary signals $\mathcal{M}$ corresponds to the chirp mass of the binary [36]. For signals that do not originate from coalescing binaries and glitches, $\mathcal{M}$ takes on unphysical values. In the unmodeled cWB analysis, the parameter $\mathcal{M}$ is used to distinguish between events with different time-frequency evolution. By selecting events with $\mathcal{M}>1 \mathrm{M}_{\odot}$ we identify a broad class of events with a chirping time-frequency signature, which includes a subclass of coalescing binary signals. The events selected by this test that also have a residual energy $E_{n}$ consistent with Gaussian noise are placed in the search class $C 3$. All other events, not included in the $C 1$ or $C 3$ class, are placed in the search class $C 2$. The union of all three independent search classes covers the full parameter space accessible to the unmodeled cWB search. 


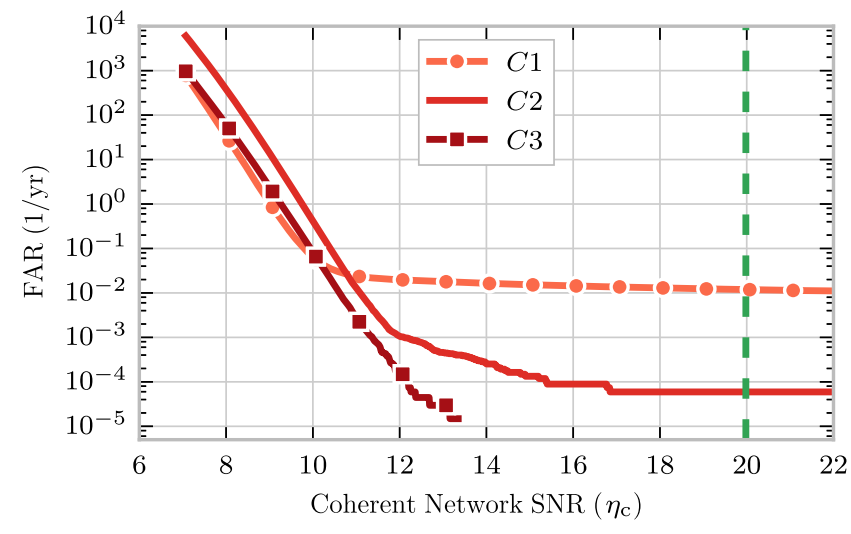

FIG. 1. Cumulative rate distribution of background events as a function of the detection statistic $\eta_{c}$ for the three $\mathrm{cWB}$ search classes. Vertical dashed line shows the value of the detection statistic for the GW150914 event.

\section{False alarm rate}

To establish the distribution of background events, we use the time-shift procedure discussed in Sec. II, using all the data available for each detector. The effective background live time for this analysis is 67400 years, obtained by analyzing more than $1.6 \times 10^{6}$ time-shifted instances of 16 days of the observation time. Figure 1 reports the cumulative false alarm rate distributions as a function of the detection statistic $\eta_{c}$ for the three defined search classes. The significance of a candidate event is measured against the background of its class. As shown in the plot, the $C 1$ search class is affected by a tail of blip glitches with the false alarm rate of approximately $0.01 y^{-1}$. Confining glitches in the $C 1$ class enhances the search sensitivity to gravitational-wave signals falling in the $C 2$ and $C 3$ classes. In fact, the tail is reduced by more than two orders of magnitude in the $C 2$ search class. The background rates in the $C 3$ search class are almost ten times lower than in $C 2$, with no prominent tail of loud events, indicating that it is highly unlikely for detectors to produce coherent background events with a chirping time-frequency evolution.

To check the homogeneity and stability of background rates shown in Fig. 1, these distributions have been compared between instances of background data, generated with different time shifts between the detectors, finding no evidence for any dependence on the time-shift interval or on the time period of data collection.

\section{Significance of GW150914 event}

GW150914 was detected with $\eta_{c}=20$ and belongs to the $C 3$ class. Its $\eta_{c}$ value is larger than the detection statistic of all observed cWB candidates. Also Fig. 2 (left) shows that the GW150914 $\eta_{c}$ value is larger than the detection statistic of any background event in its search class in 67400 years of the equivalent observation time. All other observed event candidates (orange squares) are consistent with the background.

The GW150914 significance is defined by its false alarm rate measured against the background in the $C 3$ class. Assuming that all search classes are statistically independent, this false alarm rate should be increased by a conservative trials factor equal to the number of classes. By taking into account the trials factor of 3, the estimated GW150914 false alarm rate is less than one event in 22500 years. The probability that the 16 days of data would yield a noise event with this false alarm rate is less than $16 /(365 \times 22500)=2 \times 10^{-6}$.

The union of the $C 2$ and $C 3$ search classes represents a transient search with no assumptions on the signal timefrequency evolution. The result of such analysis with just two search classes $C 1$ and $C 2+C 3$ is shown in Fig. 2

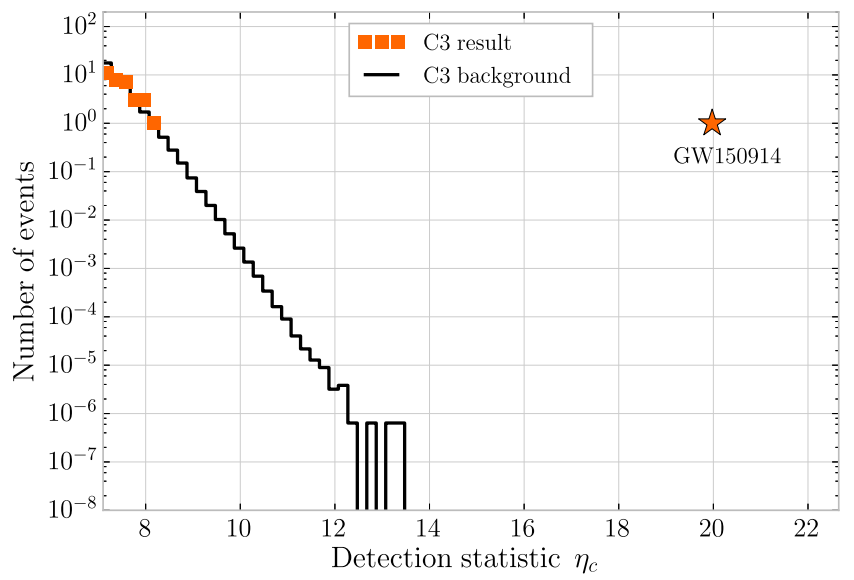

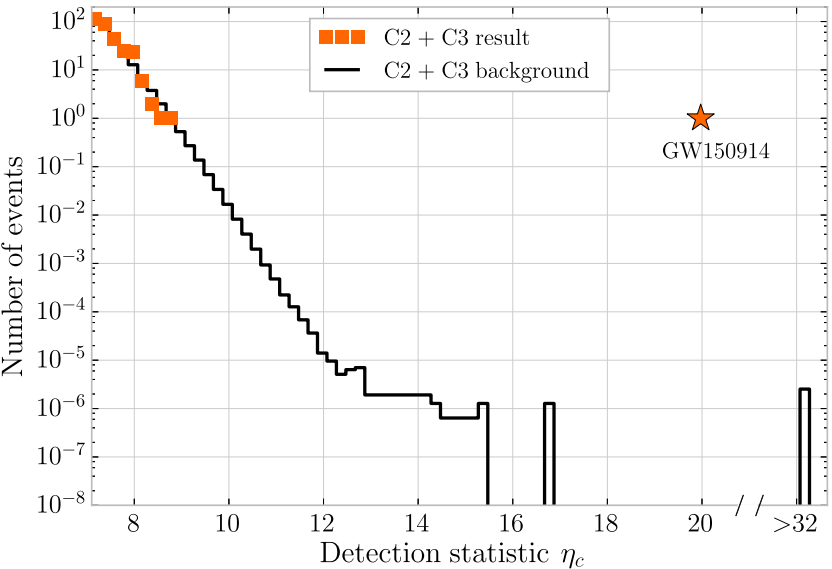

FIG. 2. Search results (in orange) and expected number of background events (black) in 16 days of the observation time as a function of the cWB detection statistic (bin size 0.2 ) for the $C 3$ search class (left) and $C 2+C 3$ search class (right). The black curve shows the total number of background events found in 67400 years of data, rescaled to 16 days of observation time. The orange star represents GW150914, found in the $C 3$ search class. 
(right). In this case there are four events louder than GW150914 in the $C 2+C 3$ class. With the trials factor of 2 , the false alarm rate is one event in 8400 years. The four loud events are produced by a random coincidence of multiple blip glitches: two nearby blip glitches in one detector and a single blip glitch in the second detector. The algorithmic test that identifies blip glitches was not designed to capture multiple ones and, therefore, missed these events.

\section{B. oLIB}

The oLIB search [23] is a search pipeline for gravitational-wave bursts designed to operate in low latency, with results typically produced in around $30 \mathrm{~min}$. However, the pipeline can operate in two modes, online and off-line. The online version identified GW150914 independently of cWB. The off-line version is used here to establish the significance of GW150914.

\section{1. oLIB pipeline overview}

The oLIB pipeline follows a hierarchical scheme, first performing a coincident event down selection followed by a fully coherent Markov chain Monte Carlo Bayesian analysis.

In the first step of the pipeline, a time-frequency map of the single-interferometer strain data from all detectors is produced using the Q-transform [37] implemented in Omicron [38]. Stretches of excess power are flagged as triggers. Neighboring triggers that occur within $100 \mathrm{~ms}$, with an identical central frequency $f_{0}$ and quality factor $Q$, are clustered together. After applying data quality vetoes as described in Sec. II, a list of triggers that fall within a $10 \mathrm{~ms}$ coincidence window (compatible with the speed-of-light baseline separation of the detectors) is then compiled.

In the second step of the pipeline, all coincident triggers identified in the first step are analyzed using LIB, a Bayesian parameter estimation and model selection algorithm that coherently explores the signal parameter space with the nested sampling algorithm [39] available in the LALInference software library [40].

LIB models signals and glitches by a single sineGaussian wavelet. Signals have a coherent phase across detectors, while glitches do not. Using this model, LIB calculates two Bayes factors, each of which represents an evidence ratio between two hypotheses: coherent signal vs Gaussian noise (BSN) and coherent signal vs incoherent glitch (BCI). These two Bayes factors are then combined into a scalar likelihood ratio $\Lambda$ for the signal vs noise (Gaussian or glitch) problem. More precisely, $\Lambda$ is obtained from the ratio of the probability distributions for the Bayes factors BSN and BCI estimated empirically from "training" sets of events. Those sets consists of $\simeq 4000$ simulated gravitational-wave signals from a uniform-in-volume source distribution and $\simeq 150$ background triggers obtained from time-shifted data for the signal and noise cases, respectively.

The final ranking statistic $\Lambda$ is evaluated for a different set of background triggers from time-shifted data in order to map a given value of the likelihood ratio into a FAR.

\section{2. oLIB analysis of GW150914}

For the purpose of this analysis, Omicron runs over the 32-1024 Hz bandwidth and selects triggers that exceed a SNR threshold of 6.5. LIB uses the following priors: uniform in sky location, uniform in central frequency $f_{0}$ in the selected bandwidth, and uniform in quality factor $Q$ from 0.1-110. Events with $\mathrm{BSN}$ or $\mathrm{BCI} \leq 0$ are discarded. We retain events with $48 \leq \tilde{f}_{0} \leq 1020 \mathrm{~Hz}$ and $2 \leq \tilde{Q} \leq 109$, where $\tilde{f}_{0}$ and $\tilde{Q}$ are median values computed from the posterior distributions delivered by LIB. The selection cut on $\tilde{Q}$ is analogous to those used by cWB to reject blip glitches and narrow-band features. The ranking statistic $\Lambda$ and its background distribution from which the FAR is deduced are computed from the training and background sets after applying all those cuts.

Because oLIB is able to run on short data segments $(\gtrsim 3 \mathrm{~s})$, this search analyzed nearly all available data, which amounted to 17.4 days, i.e., $~ 10 \%$ more coincident data than cWB. The data were time-shifted in 1-sec intervals to produce the equivalent of 106000 years of background data. The background distribution is plotted as a function of $\log \Lambda$ in Fig. 3. As shown in the same figure GW150914 has a ranking statistic of $\log \Lambda=0.80$, corresponding to a FAR of roughly 1 in 27000 years. It is the only event in the search results satisfying the selection cuts.

\section{BayesWave follow-up}

The BayesWave pipeline is a Bayesian algorithm designed to robustly distinguish GW signals from glitches in the detectors $[31,41]$. In this search, BayesWave is run as

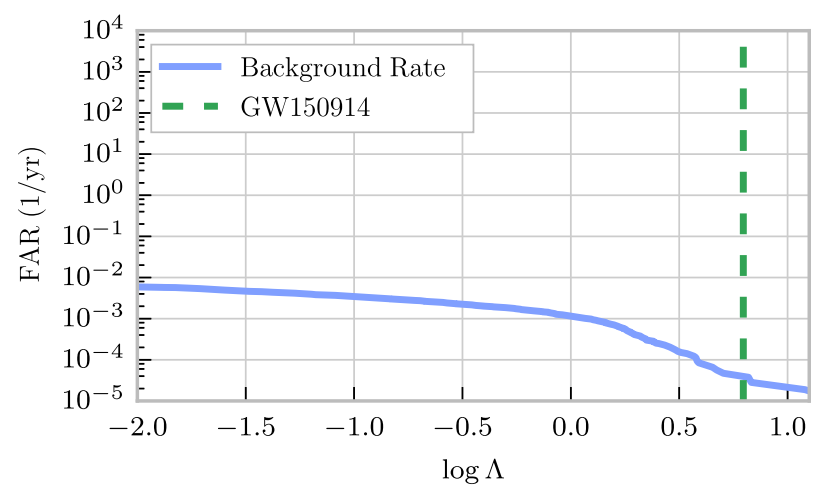

FIG. 3. Cumulative rate distribution of background events as a function of oLIB ranking statistic $\log \Lambda$. GW150914 is the only event in the search results to pass all thresholds. Its statistic value $\log \Lambda=0.80$ corresponds to a background FAR of $\simeq 1$ in 27000 years. 
a follow-up analysis to triggers identified by cWB. For each candidate event, BayesWave compares the marginalized likelihood, or evidence, among three hypotheses: the data contain only Gaussian noise, the data contain Gaussian noise and noise transients (glitches), or the data contain Gaussian noise and an astrophysical signal.

The BayesWave algorithm models signals and glitches using a linear combination of sine-Gaussian wavelets. The number of wavelets needed in the glitch or signal model is not fixed a priori, but instead is optimized using a reversible jump Markov chain Monte Carlo. The glitch model fits the data separately in each interferometer with an independent linear combination of wavelets. The signal model reconstructs the candidate event at some fiducial location (the center of the Earth), taking into account the response of each detector in the network to that signal. BayesWave uses a parametrized phenomenological model, BayesLine, for the instrument noise spectrum, simultaneously characterizing the Gaussian noise and instrument/ astrophysical transients [41].

BayesWave produces posterior distributions for the parameters of each model under consideration. For the signal model, this includes the waveform, as constructed from sums of sine-Gaussian wavelets, and the source position. Waveform reconstructions are used to produce posterior distributions for characteristics such as the duration, central frequency, and bandwidth of the signal, which are used to compare the data to theoretical models. The marginalized posterior (evidence) for each model is calculated by marginalizing over the different dimension waveform reconstructions, and then is used to rank the competing hypotheses.

BayesWave is used as a follow-up analysis for candidate events first identified by cWB. The combined cWB + BayesWave data analysis pipeline has been shown to allow high-confidence detections across a range of waveform morphologies [21,22]. The cWB + BayesWave pipeline uses the Bayes factor, comparing the signal and glitch models $\left(\mathcal{B}_{S G}\right)$ as its detection statistic. Bayes factors are reported on a natural logarithmic scale $\ln \mathcal{B}_{S G}$, which scales with $N \ln \mathrm{SNR}$, where $N$ is the number of wavelets used in the reconstruction [22]. The consequence is that BayesWave assigns a higher detection statistic to signals with nontrivial time-frequency structure. Though Bayes factors used by Bayeswave and oLIB methods both produce a measure of coherence between the signal morphologies observed in multiple detectors, the above calculation indicates that BayesWave, $\mathcal{B}_{S G}$, also includes a measure of the signal complexity.

The "off-line" BayesWave pipeline analyzes all cWB zero-lag and background events with a detection statistic $\eta_{c}>11.3$ and correlation coefficient $c_{c}>0.7$. The threshold on $\eta$ for event follow-up is a compromise between computational cost and in-depth analysis of cWB events. The BayesWave computation is performed over a $4-\mathrm{sec}$

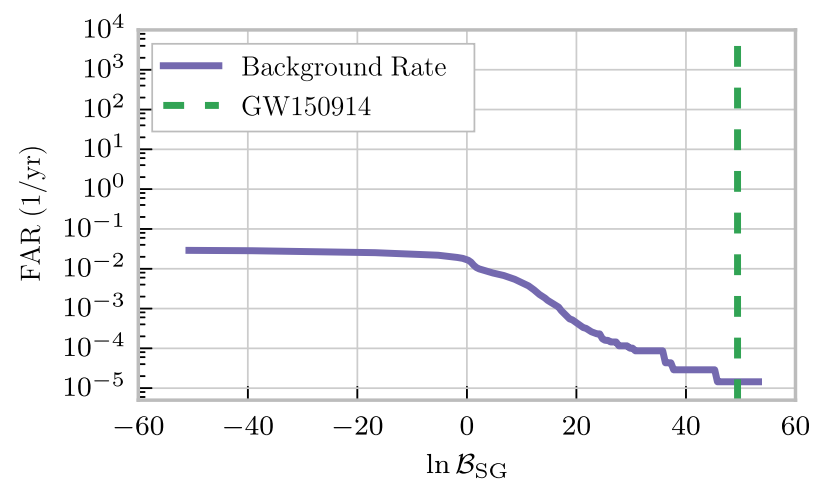

FIG. 4. Cumulative rate distribution of background events as a function of the cWB + BayesWave detection statistic $\ln \mathcal{B}_{S G}$. The $\mathrm{cWB}+$ BayesWave pipeline considers all cWB candidates with $\eta_{c}>11.3$ (combining all three curves in Fig. 1). In the equivalent of 67400 years of data, GW150914 was the only zero-lag event to pass all thresholds. Only one noise coincidence is ranked higher than GW150914.

segment of data ${ }^{2}$ centered on the event time reported by cWB. We use $1 \mathrm{sec}$ of data around the event time for model comparison, while the remainder of the segment is used for spectral estimation. We perform the analysis in the Fourier domain over the frequency range of $32<f<1024 \mathrm{~Hz}$ though, for cWB candidates with central frequency $f_{\mathrm{cWB}}<$ $200 \mathrm{~Hz}$ (including GW150914), BayesWave used a maximum frequency of $512 \mathrm{~Hz}$ to reduce the computational cost of the analysis. Both the signal and the glitch model require at least one wavelet (to make them disjoint from one another and the Gaussian noise model) and have a maximum of 20 wavelets allowed in the linear combination. Most of the priors used in the analysis are as described in [31] and [22], with the following changes. The prior on the "quality factor" of the wavelets $Q$ has been extended to include lower values, so that it is uniform over the interval $[0.1,40]$. The low $Q$ values allow blip glitches to be correctly characterized with a small number of wavelets. Also, the functional form of the glitch amplitude prior has been modified to scale as a power law rather than an exponential in the large SNR limit. The new prior better reflects the belief that very loud events $($ SNR $>100)$ are more likely to be glitches than signals.

Figure 4 shows the cumulative rate distribution of background events as a function of the cWB + BayesWave detection statistic $\ln \mathcal{B}_{S G}$. The $\mathrm{cWB}+$ BayesWave pipeline considers the triggers from all cWB search classes together (all curves in Fig. 1) as a single search. The explicit glitch model used by BayesWave reduces the tail in the background distribution [22], so that loud background events are down-weighted rather than grouped into different classes. In

\footnotetext{
${ }^{2}$ The $4 \mathrm{sec}$ segment length was shown in testing to be the minimum amount of data needed to estimate the power spectral density.
} 
TABLE I. Summary of the BBH simulations used for estimating search efficiency.

Total mass $M=m_{1}+m_{2}$

Mass ratio, $q=m_{2} / m_{1}$

Spin magnitude $\left|\mathbf{a}_{1,2}\right|$

Waveform model

$30-150 M_{\odot}$

$0.25,0.5,1.0$

$0-0.99$

SEOBNRV2

the equivalent of 67400 years of $\mathrm{O} 1$ data, $2374 \mathrm{cWB}$ events warranted a BayesWave follow-up and only one noise coincidence $\left(\ln \mathcal{B}_{S G}=53.1 \pm 3.4\right)$ was ranked higher than GW150914 $\left(\ln \mathcal{B}_{S G}=49.4 \pm 0.8\right)$. GW150914 is the only zero-lag event to pass all thresholds. Investigations of the highest ranking background events have revealed remarkably similar glitches in the two detectors which, were it not for the large, unphysical time shifts applied to the data, would be indistinguishable from a GW signal. However, the waveform morphology of the most significant background events is in no way similar to a BBH merger signal. Treating all cWB candidates as coming from the same search, BayesWave estimates a FAR for GW150914 of 1 in 67400 years.

\section{SEARCH SENSITIVITY}

In this section, we demonstrate the ability of transient searches to detect GWs from BBH mergers. We use simulated gravitational waveforms that cover all three phases of BBH coalescence, i.e., inspiral, merger and ringdown. The analysis is performed by adding simulated $\mathrm{BBH}$ waveforms to the detector data, and recovering them using the three burst pipelines described in Sec. III.

\section{A. Simulation data set}

BBH systems are characterized by the masses $m_{1}$ and $m_{2}$, dimensionless spin vectors $\mathbf{a}_{1}$ and $\mathbf{a}_{2}$ of the two component black holes, the source distance $D$, its sky-location coordinates, and the inclination of the $\mathrm{BBH}$ orbital momentum vector relative to the line of sight to Earth. The black hole spins are obtained from the dimensionless spin vectors by $\mathbf{S}_{i}=m_{i}^{2} \mathbf{a}_{\mathbf{i}}$, where $\left|\mathbf{a}_{i}\right| \leq 1$.

The simulation includes binaries that are isotropically located on the sky and isotropically oriented, with total masses $M=m_{1}+m_{2}$ uniformly distributed between 30 and $150 \mathrm{M}_{\odot}$, that is within a factor of $\sim 2$ of the estimated total mass for GW150914 [29]. We generate three separate sets, each with a fixed mass ratio $q=m_{2} / m_{1} \in\{0.25,0.5,1.0\}$. We assume that the black hole spins are aligned with the binary orbital angular momentum, with a spin magnitude uniformly distributed across $\left|\mathbf{a}_{1,2}\right| \in[0,0.99]$. The distances are drawn from distributions within $3.4 \mathrm{Gpc}$ such that we get good sampling for a range of SNR values around the detection threshold. The simulation does not include redshift corrections, which introduces small systematic errors for the more distant sources. The signals are distributed uniformly in time with a gap of $100 \mathrm{sec}$ between them.

The BBH waveforms analyzed in this study have been generated using the SEOBNRV2 model in the LAL software library $[42,43]$. This model only accounts for the dominant $\ell=2, m=2 \mathrm{GW}$ radiated modes. The waveforms are generated with an initial frequency of $15 \mathrm{~Hz}$. The data sets are summarized in Table I.

\section{B. Results}

To quantify the results of the study, we use the sensitive radius which is the radius of the sphere with volume $V=\int 4 \pi r^{2} \epsilon(r) d r$, where $\epsilon(r)$ is the averaged search efficiency for sources at distance $r$ with random sky position and orientation [16]. For each pipeline, we calculate the sensitive radius as a function of FAR. The results are shown in Fig. 5. For example, at a FAR of 1 per thousand years, the three searches show similar performance, with each detecting the simulated equal-mass BBH population to a sensitive distance in the range 700 to

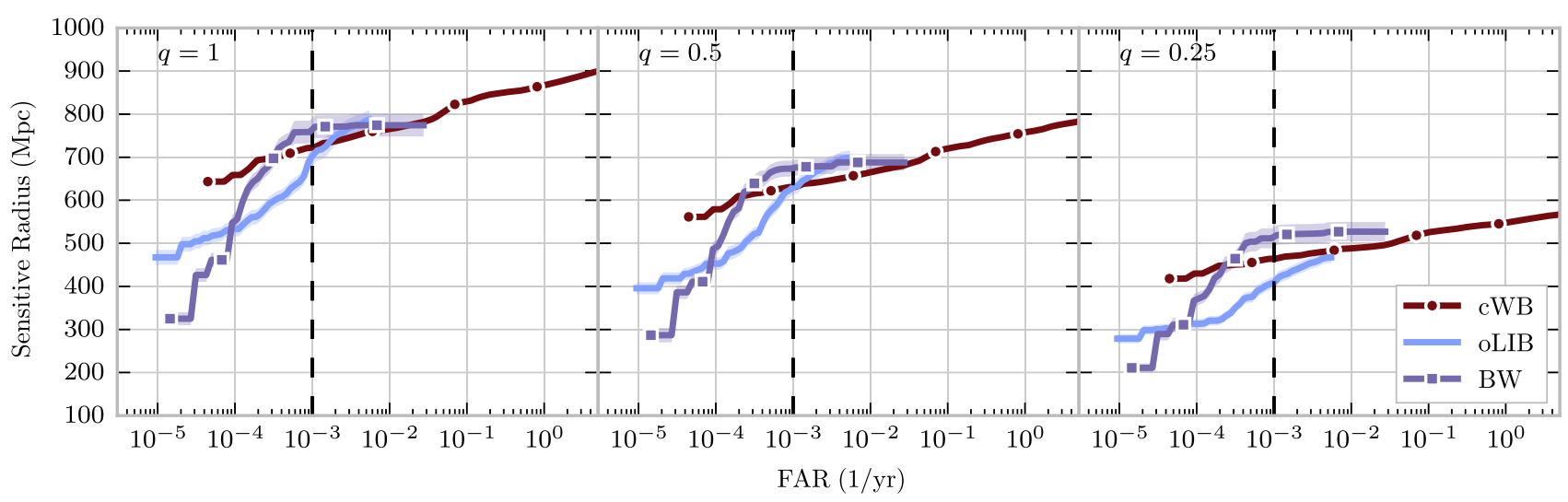

FIG. 5. Sensitive radius for the different search pipelines for simulated BBH waveforms with different mass ratios $q$. The sensitive radius measures the average distance to which the search detects with a given FAR threshold. The cWB results include all three search classes, with a corresponding trials factor. 


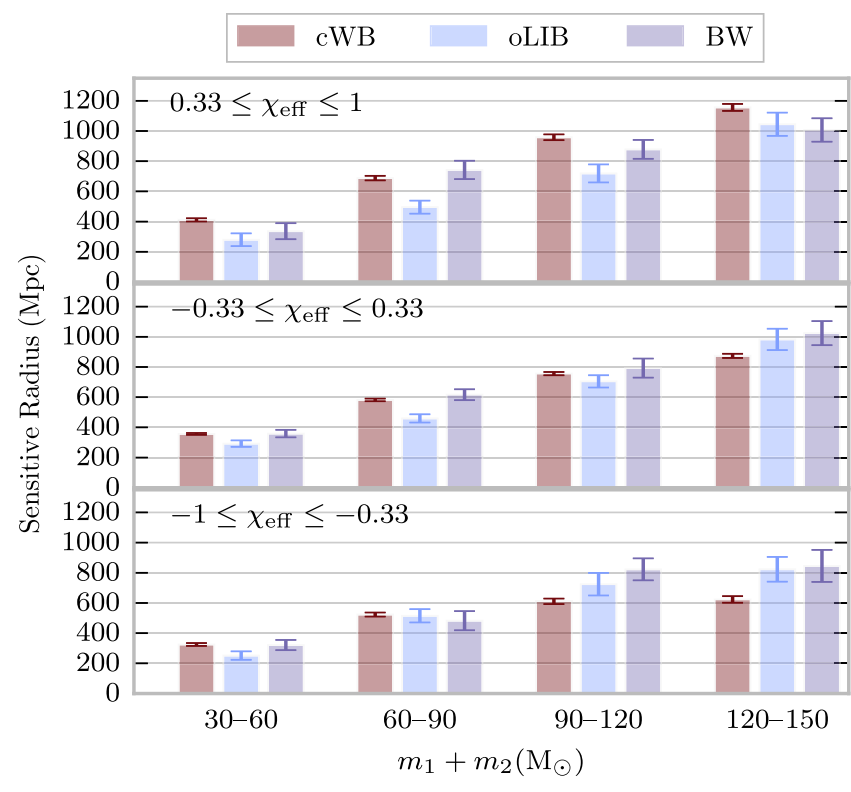

FIG. 6. Dependence of sensitive radius on spins of BBH. To investigate the effect of spins of black holes on the detection of BBH systems, we show the search radius $R$ for each pipeline for varying effective spins with mass ratio $q=1$ at $\mathrm{FAR}=$ $10^{-3} 1 / \mathrm{yr}$. The total mass range is varied from $30-150 \mathrm{M}_{\odot}$, while the effective spin is distributed into three bins: aligned spins $\left(\chi_{\text {eff }} \in[0.33,1]\right)$, antialigned $\left(\chi_{\text {eff }} \in[-1,-0.33]\right)$ and nonspinning $\left(\chi_{\text {eff }} \in[-0.33,0.33]\right)$. The error bars represent the statistical uncertainty of the sample. The cWB results include all three search classes, with a corresponding trials factor.

$800 \mathrm{Mpc}$. To the far left side of the plots (very low FAR), the differences between pipelines are dominated by the loudest few background events; the cWB $C 3$ search class selection for chirping events allows many BBH signals to be recovered with very low FAR.

The effect of intrinsic BBH parameters (component masses and spins) on the sensitive radius of the three pipelines is summarized in Fig. 6. The three panels of the figure correspond to three bins of effective spin. Effective spin is defined as in [29]: $\chi_{\text {eff }}=\left(\frac{\mathbf{S}_{1}}{m_{1}}+\frac{\mathbf{S}_{2}}{m_{2}}\right) \cdot \frac{\hat{\mathbf{L}}}{M}$, with $\hat{\mathbf{L}}$ the direction of orbital angular momentum. Depending on the mass and spin of the binary, the sensitive radius can vary from about $250 \mathrm{Mpc}$ up to over $1 \mathrm{Gpc}$. Over this range, larger masses are detectable to further distances. Spins which are aligned with the orbital angular momentum tend to increase the sensitive radius, while antialigned spins make the systems more difficult to detect. For the mass/spin bin most like GW150914, 60-90 $\mathrm{M}_{\odot}$, the sensitive radius of the searches is between 400 and $600 \mathrm{Mpc}$.

\section{SOURCE CHARACTERIZATION}

In [29], we present estimates for the parameters of the binary black hole model that best describes GW150914. These parameters include the masses and spins of the binary components, and their posterior distributions represent our most complete description of the astrophysical source. In this work, we take a complementary approach, by using the outputs of the burst pipelines described in Sec. III to characterize the event. Many of the burst pipeline outputs are available in low latency, so this approach can inform follow-up studies in a timely fashion. For example, the cWB estimate of the GW150914 chirp mass was available within minutes, and provided the first evidence that this signal originated from merging black holes. Likewise, low-latency position estimates are used for counterpart searches [28].

Burst analyses are also able to estimate the time evolution of observed waveforms, a process we refer to as waveform reconstruction. Burst waveform reconstruction algorithms do not rely on astrophysical models. Instead, estimates of the coherent gravitational-wave power observed by the detector network are used to reconstruct the signal. These waveform reconstructions are valuable: they provide an unbiased view of the signal most consistent with the observatory data. Such reconstructed signals can be used to classify the source type, compare with models, and potentially identify unexpected features. In this section, we present how the outputs of the burst pipelines were used to estimate the source position, reconstruct the waveform, and characterize the $\mathrm{BBH}$ source. We also compare the reconstructed waveforms with a set of numerical relativity waveforms, in order to check the consistency of our results against the most precise class of models available.

\section{A. Source localization}

Three burst algorithms (cWB, BayesWave, and LIB) produce localization estimates for the GW event. These "skymaps" can be interpreted as the posterior probability distribution of the source's right ascension $(\alpha)$ and declination $(\delta)$ given the observed data. cWB produces skymaps during its detection process by maximizing a constrained likelihood on a grid over the sky; these are available within minutes of the candidate's detection. LIB and BayesWave perform more computationally expensive analyses, and so produce results with higher latency. LIB uses a space of single sine-Gaussian waveforms as its waveform model, and produces skymaps after 1 to $2 \mathrm{~h}$, whereas BayesWave maps can take as long as several days to be produced, since it explores a larger parameter space of superpositions of sine-Gaussian waveforms. Each algorithm makes different and somewhat complementary assumptions about the signal, and these assumptions affect their localization estimates. By localizing signals with multiple algorithms, we can cross-check and validate the localization estimate and identify any systematic difference between the algorithms [44].

An overview of the skymaps used by astronomers to search for counterparts to GW150914 may be found in [28], including the cWB and LIB skymaps. Here, we 
compare cWB, LIB, and BayesWave skymaps in addition to the map produced by LALInference with binary coalescence templates, which samples the posterior distribution of all signal parameters using signal waveforms that cover the inspiral, merger and ringdown phase [40]. For GW150914, we expect the LALInference map to yield a relatively precise localization, because it assumes a waveform from a compact binary coalescence, instead of the broad waveform classes used by the burst pipelines. Burst localization algorithms produce systematically larger skymaps than template-based algorithms because they make fewer assumptions about the waveform. However, the
LALInference map reported here also includes the effects of calibration uncertainty within the detectors, which significantly widen the uncertainty of this reconstruction [45]. In principle, calibration effects could also be included in the burst skymaps, but what is shown here represents the information that was available at the time electromagnetic astronomy observations began [28].

Figure 7 shows Mollweide projections in $(\alpha, \delta)$ of all skymaps considered, as well as overlays of the $50 \%$ and $90 \%$ contours in a rotated frame of reference. Figure 8 shows the marginal distributions for the polar angle from the line-of-sight between the two LIGO detectors. This
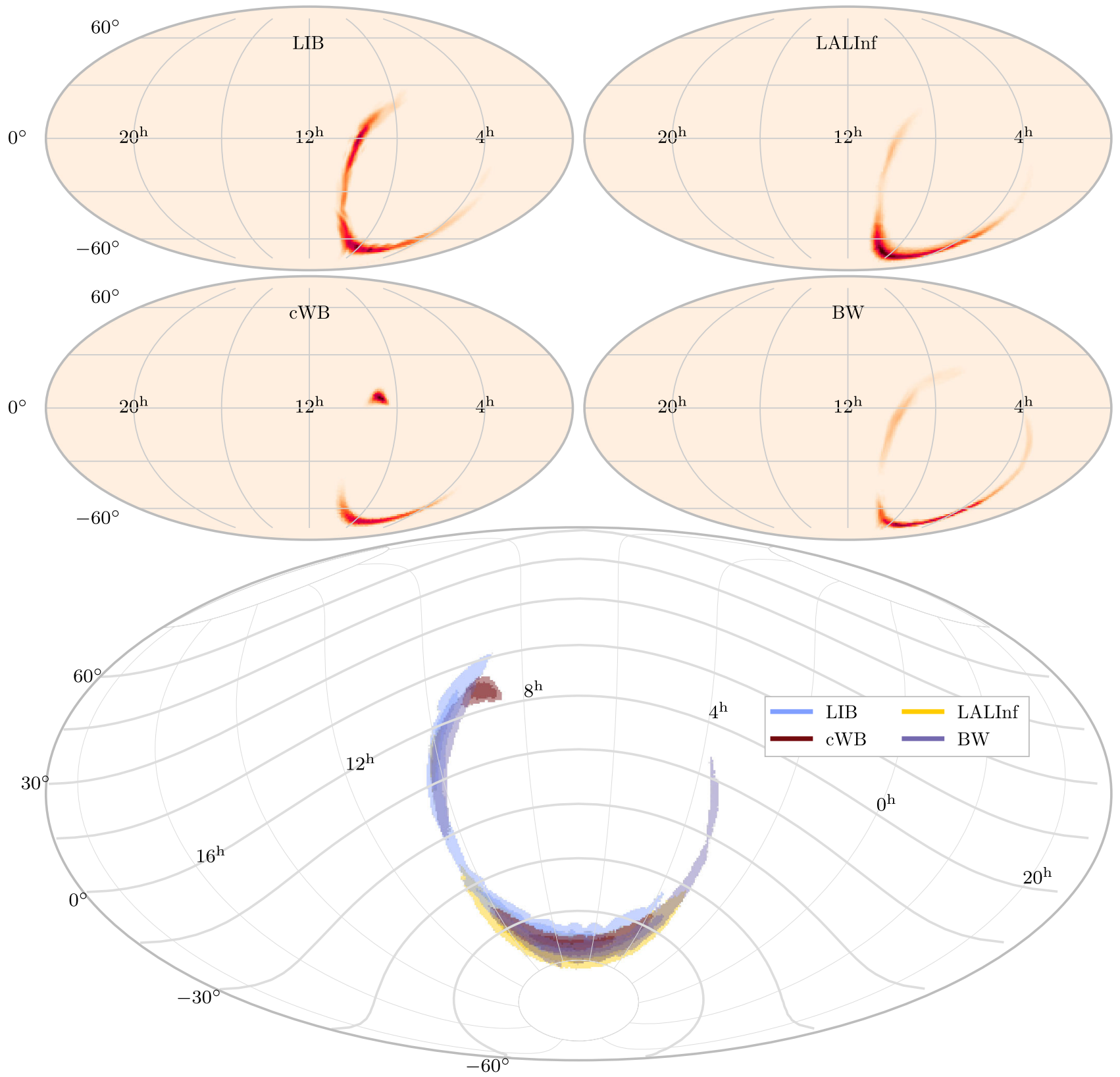

FIG. 7. All-sky projections of several skymaps produced for GW150914. Above, each map is shown by itself in celestial coordinates. Below, a rotated coordinate system shows contours defining the $50 \%$ and $90 \%$ confidence regions for four reconstructions. 


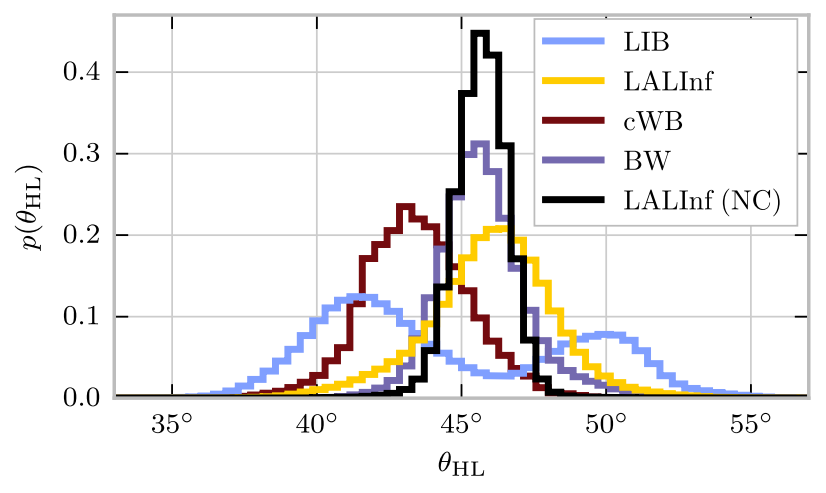

FIG. 8. Marginal distributions of the polar angle defined by triangulation. These give a measure of the width of each ring.

marginal distribution captures the width of the triangulation rings. All maps are consistent with some differences due to the reconstruction algorithms. For example, the cWB map has a "northern island" near the equator not seen in other maps. The shape and placement of the island is affected by the LIGO detector responses at this particular sky location $[28,44]$. The Hanford-Livingston network is sensitive to only one polarization through most of the sky, and cWB uses this to constrain the reconstructed signal, with the exception of regions like the island where the network sensitivity is comparable for both polarizations. In this case, cWB relaxes the constraint and cannot break a degeneracy between sky locations near the island. We note this occurs only when the triangulation ring falls near one of these regions and may not be present for other events.

To measure the similarity between the skymaps, Table II presents the fidelity $F(p, q)=\sum_{i} \sqrt{p_{i} q_{i}} \in[0,1]$ for the various algorithms considered, where $p_{i}$ and $q_{i}$ are the probability densities assigned to pixels at the same coordinates in two different skymaps. $F$ is closer to one if the maps are more similar and $F$ closer to zero if the maps are dissimilar. For comparison, we also include a skymap produced by LALInference that does not include calibration uncertainties. This similarity measurement is between $28 \%$ and $87 \%$ for different pairs of skymaps. To check the

TABLE II. Confidence regions and fidelity values from GW150914. The fidelity measures the similarity of two skymaps. The LALInference skymaps are shown both with (LALInf) and without (LALNoCE) calibration uncertainty included. The shown burst skymaps do not include calibration uncertainties, which would make the uncertainty regions larger.

\begin{tabular}{lcccccc}
\hline \hline & \multicolumn{2}{c}{ Confidence regions } & \multicolumn{4}{c}{ Fidelity } \\
& $50 \%$ & $90 \%$ & LIB & BW & LALInf LALNoCE \\
\hline cWB & $98 \mathrm{deg}^{2}$ & $308 \mathrm{deg}^{2}$ & 0.55 & 0.55 & 0.51 & 0.50 \\
LIB & $208 \mathrm{deg}^{2}$ & $746 \mathrm{deg}^{2}$ & $\ldots$ & 0.45 & 0.68 & 0.28 \\
BW & $101 \mathrm{deg}^{2}$ & $634 \mathrm{deg}^{2}$ & $\ldots$ & $\ldots$ & 0.68 & 0.87 \\
LALInf & $150 \mathrm{deg}^{2}$ & $610 \mathrm{deg}^{2}$ & $\ldots$ & $\ldots$ & $\ldots$ & 0.81 \\
LALNoCE & $48 \mathrm{deg}^{2}$ & $150 \mathrm{deg}^{2}$ & $\ldots$ & $\ldots$ & $\ldots$ & $\ldots$ \\
\hline \hline
\end{tabular}

robustness of the parameter estimation results, we simulated 29 transients with waveforms similar to GW150914, generated using the SEOBNRv2 approximant $[42,43]$, by actuating on the mirrors at the end of the $4 \mathrm{~km} \mathrm{LIGO} \mathrm{arms.}$ We repeat the analysis on each of these hardware injections. We find similar fidelity measurements as with the GW150914 event, suggesting that this level of agreement between the algorithms is typical for BBH waveforms at the SNR of GW150914.

\section{B. Waveform reconstruction}

To extract the astrophysical signal from detector noise, we reconstruct waveforms whose projection onto both the $\mathrm{H} 1$ and $\mathrm{L} 1$ detectors is consistent with the data. The cWB algorithm [35] performs waveform reconstruction using a constrained maximum likelihood approach (see Sec. III A 1). BayesWave [31,41] uses a variable dimension continuous wavelet basis to produce a posterior distribution for the gravitational waveform present in a data set. In contrast to analyses based on compact object merger templates, which attempt to find the best fit parameters within a well-defined waveform family, the cWB and BayesWave waveform reconstruction algorithms make very weak assumptions about the form of the signal. The oLIB pipeline assumes a sine-Gaussian waveform, and so provides a less detailed reconstruction. The BayesWave version used in this analysis assumes that the signal is elliptically polarized, but is otherwise free to reconstruct any astrophysical signal in the searched time-frequency volume.

Figure 9 shows both the cWB point estimate and the BayesWave 90\% credible interval for the reconstructed, whitened, time-domain signal waveform, as projected onto each detector. The waveforms are seen to largely agree, and include the main expected features from a chirp signal due

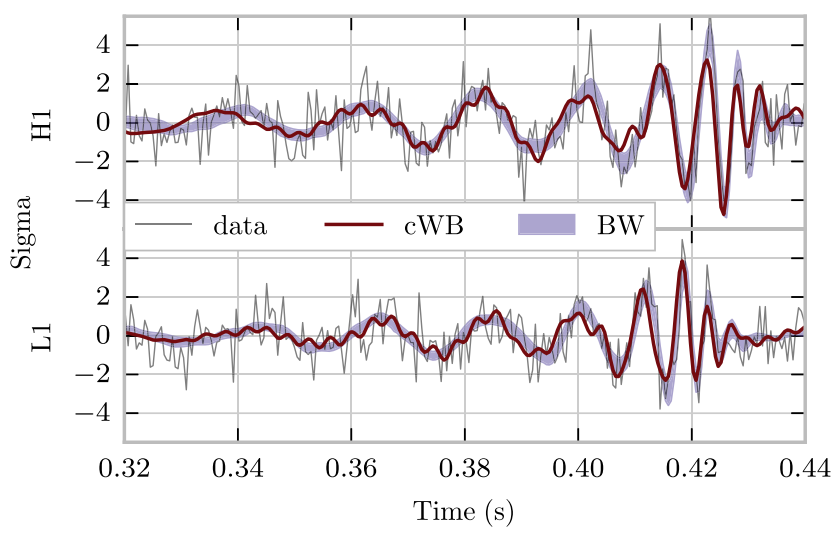

FIG. 9. The cWB point estimate for the waveform and the $90 \%$ credible interval from the BayesWave analysis. The reconstructed waveforms and shown data are whitened using estimated noise curves for each detector at the time of the event. On the y-axis, sigma is a measure of the amplitude in terms of the number of noise standard deviations. 
OBSERVING GRAVITATIONAL-WAVE TRANSIENT ...

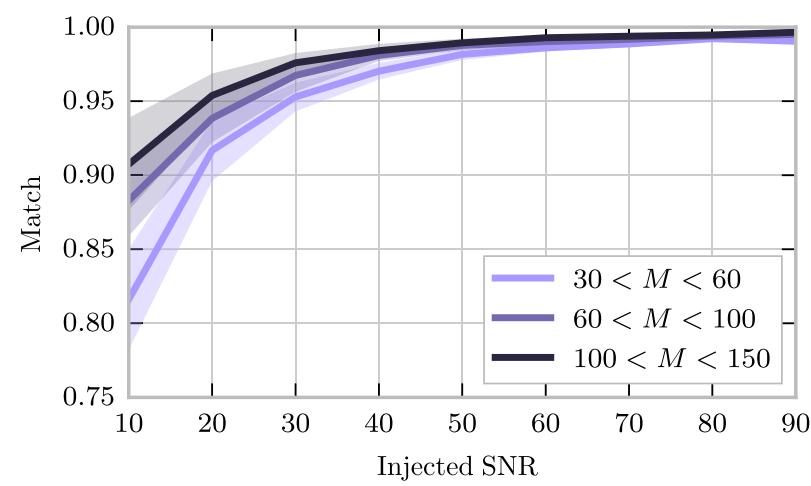

FIG. 10. Match between the whitened injected and BayesWave reconstructed waveforms for the simulation set described in Sec. IV. The line indicates the median match and the shaded region shows the $1 \sigma$ uncertainty. $M$ indicates the total mass of the black hole binary, measured in solar masses.

to a compact object merger. The BayesWave waveforms have a median match of $94 \%$ with the posterior samples from a Bayesian analysis that uses waveform templates that account for the inspiral, merger and ringdown phases of the $\mathrm{BBH}$ coalescence [40].

To measure the accuracy of these reconstructions, we use the set of simulated BBH systems described in Sec. IV. For each event recovered by BayesWave, we calculate the match between the injected and reconstructed waveforms. The results are shown in Fig. 10. At fixed SNR the match between the simulated and the reconstructed waveform is systematically higher for higher mass signals because larger mass BBH signals have a smaller time-frequency volume, allowing them to be fit with a smaller number of wavelets. For the simulations similar to GW150914, in the mass bin from 60 to $100 \mathrm{M}_{\odot}$ and around network SNR 20, we see most matches are between $90 \%$ and $95 \%$ accurate.

\section{Parameter estimation with generic signal features}

The source parameters of GW150914, such as component masses and spins, can be well characterized by using an analytical model of BBH signals to compute their posterior distributions [29]. Here, we take a different approach, which uses the outputs of the burst pipelines to provide a coarse estimate of the model parameters. The BayesWave and cWB waveform reconstructions can be used to compute a variety of parameters that summarize the signal, such as the central frequency, duration and bandwidth. These parameters can then be used to help identify characteristics of the astrophysical system that generated the signal. Using waveform templates for a BBH merger, we can derive predictions for the central frequency and bandwidth of the signal in each detector as a function of the mass, mass ratio and spins. Figure 11 shows the posterior distribution for the central frequency and bandwidth derived from the BayesWave analysis of GW150914,
PHYSICAL REVIEW D 93, 122004 (2016)

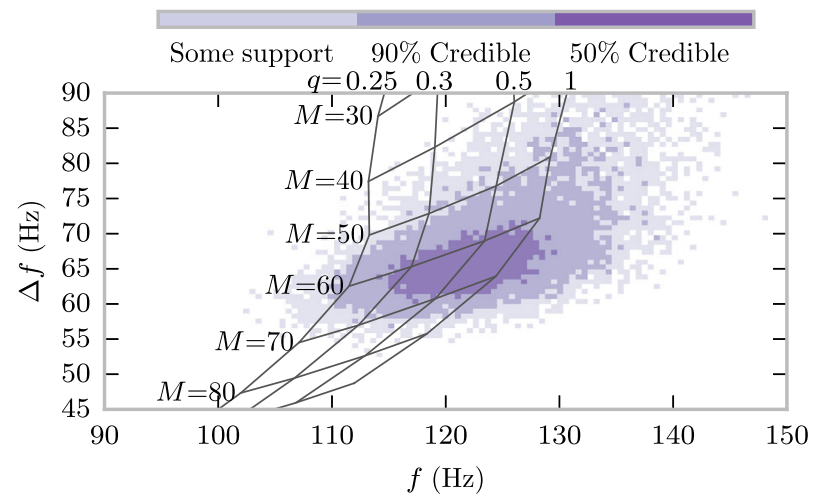

FIG. 11. The posterior distributions for the central frequency and bandwidth inferred from the whitened waveform posteriors produced by BayesWave for GW150914 are compared to the values predicted by the $\mathrm{BBH}$ merger templates with zero spin and total mass $M$ (in units of solar mass) and mass ratio $q$, as indicated by the mesh of lines. The regions of high posterior probability are consistent with the best fit values of total mass and mass ratio [29].

with an overlaid grid showing the values predicted from a black hole merger model with zero spins and total mass $M$ and mass ratio $q$ as indicated. From our companion paper, [29], the best description of this signal yields a detector frame total mass of $M=71_{-4}^{+5} \mathrm{M}_{\odot}$ and a mass ratio of $q=0.82_{-0.20}^{+0.17}$. Comparing these best fit values to the regions of high posterior density shown in Fig. 11, we find that the values lie within the $90 \%$ credible interval produced using the BayesWave outputs.

Applying the same procedure to the 29 GW150914-like hardware injections we found that the central frequency and bandwidth of the injected signals fell within the 50\% credible interval $50 \%$ of the time, and within the $90 \%$ credible interval $89 \%$ of the time, showing that the analysis is consistent.

\section{Chirp mass from time-frequency signature}

The cWB pipeline obtains the time-frequency patterns of the events by using a discrete wavelet transform. Given a pattern with $N$ time-frequency components $\left(t_{i}, f_{i}\right)$, $i=1, \ldots, N$ from a coalescing binary, at the leading post-Newtonian order it is described by the time-frequency evolution [36]

$$
\frac{96}{5} \pi^{8 / 3}\left(\frac{\mathrm{G} \mathcal{M}}{c^{3}}\right)^{5 / 3} t+\frac{3}{8} f^{-8 / 3}+C=0,
$$

where $\mathcal{M}$ is the chirp mass parameter, $\mathrm{G}$ is the gravitational constant, $c$ is the speed of light and $C$ is a constant related to the merger time. By fitting this time-frequency evolution to the data $\left(t_{i}, f_{i}\right)$, we can find the mass parameter $\mathcal{M}$ [46]. For a signal from a coalescing binary with component masses $m_{1}$ and $m_{2}$ it corresponds to the chirp mass of the 
system $\mathcal{M}=\left(m_{1} m_{2}\right)^{3 / 5} /\left(m_{1}+m_{2}\right)^{1 / 5}$. The chirp mass error is estimated using a bootstrapping procedure, where multiple subsets of data points $\left(t_{i}, f_{i}\right), i=1, \ldots, N$ are randomly selected to estimate the chirp mass.

The real-time search that first detected GW150914 estimated its detector frame chirp mass to be $27.6 \pm 2.0 \mathrm{M}_{\odot}$. This result is consistent with the LALInference estimate of $30_{-2}^{+2} \mathrm{M}_{\odot}$ [29]. To check the accuracy of the real-time method, we studied 29 hardware injections with parameters similar to those inferred for GW150914. We found that this method was able to accurately reconstruct the chirp masses of these simulated signals, with a precision similar to the quoted uncertainty.

\section{E. Overlap between reconstructed waveform and BBH model}

This section presents the comparison of the reconstructed signal of the event, from BayesWave and cWB, with predictions from NR. The goal is to provide a quantitative check that the recovered signal power is consistent with a $\mathrm{BBH}$ source as predicted by numerical relativity simulations; more stringent tests of general relativity are available in [47]. By making very weak assumptions about the signal, the waveform reconstruction provides a largely model-agnostic representation of the full astrophysical signal content. In turn, the NR waveform is the direct solution to the full Einstein equations without any assumptions other than those necessary to numerically solve the equations, e.g. finite discretization and finite extraction radius. The NR waveforms used in this study were generated by the code in [48]. The errors in the phase and amplitude of the waveform that arise from these approximations are addressed in [49]. Comparing directly to NR waveforms allows us to explore regions of parameter space where the analytic templates [29] have not yet been tuned, such as highly precessing spin configurations and their higher harmonics. The study is a simple way to compare the reconstructed astrophysical signal with the predictions of general relativity with minimal assumptions. By comparing the NR waveforms, which cover regions of the parameter space which are not necessarily well modeled and include higher harmonics, with the model-independent reconstructed waveforms which can recover the full astrophysical signal content, we are sensitive to departures from both the analytic templates used elsewhere and from the predictions of general relativity. In fact, we find excellent agreement between this study and the parameter estimation performed with analytic templates, as well as with the parameter estimation procedure using only NR waveforms which is reported in [50]. We discuss these findings below.

The natural figure of merit for this comparison is the fitting factor. We define the network match between the reconstructed waveform $s_{\text {rec }}^{(d)}$ in detector $d$ and the NR waveform $h_{\mathrm{NR}}$ as [51]

$$
\mathcal{N}=\frac{\sum_{d} \max _{t_{0}, \phi_{0}}\left(s_{\mathrm{rec}}^{d} \mid h_{\mathrm{NR}}\right)_{d}}{\left[\sum_{d}\left(h_{\mathrm{NR}} \mid h_{\mathrm{NR}}\right)_{d}\right]^{1 / 2} \times\left[\sum_{d}\left(s_{\mathrm{rec}}^{d} \mid s_{\mathrm{rec}}^{d}\right)_{d}\right]^{1 / 2}} \text {. }
$$

where the sums run over the $\mathrm{H} 1$ and $\mathrm{L} 1$ detectors and $(a \mid b)_{d}$ defines the noise-weighted inner product between waveforms $a$ and $b$ for detector $d$. The fitting factor is the network match $\mathcal{N}$ maximized over the total mass and orbital inclination [52].

The reconstructed waveforms are compared to $102 \mathrm{BBH}$ waveforms that have been used previously to investigate the feasibility of detecting precession and higher order modes [48,53-61]. We also include an additional four new simulations with intrinsic parameters motivated by parameter estimation studies of GW150914 [29]. Note that the NR simulations are not a continuous representation of the parameter space, but rather a discrete set of astrophysically interesting, generic systems. Each NR waveform, $h_{\mathrm{NR}}$, is parametrized by the mass ratio $q=m_{2} / m_{1}<1$ and spin configuration of the system.

Figure 12 shows the fitting factors between BayesWave and $\mathrm{cWB}$ and the NR waveforms in terms of the mass ratio $q$ and the dot products between the component spins and the orbital angular momentum, $\mathbf{a}_{\mathbf{i}} \cdot \hat{\mathbf{L}}$ for $i=1,2$. The figure also serves to demonstrate the coverage of the parameter space by the NR simulations. We find that the parameter space of NR waveforms favored by both algorithms is similar. Specifically, nearly symmetric mass configurations and small values for $\mathbf{a}_{\mathbf{i}} \cdot \hat{\mathbf{L}}$ for both components are preferred, although the lack of variation in the

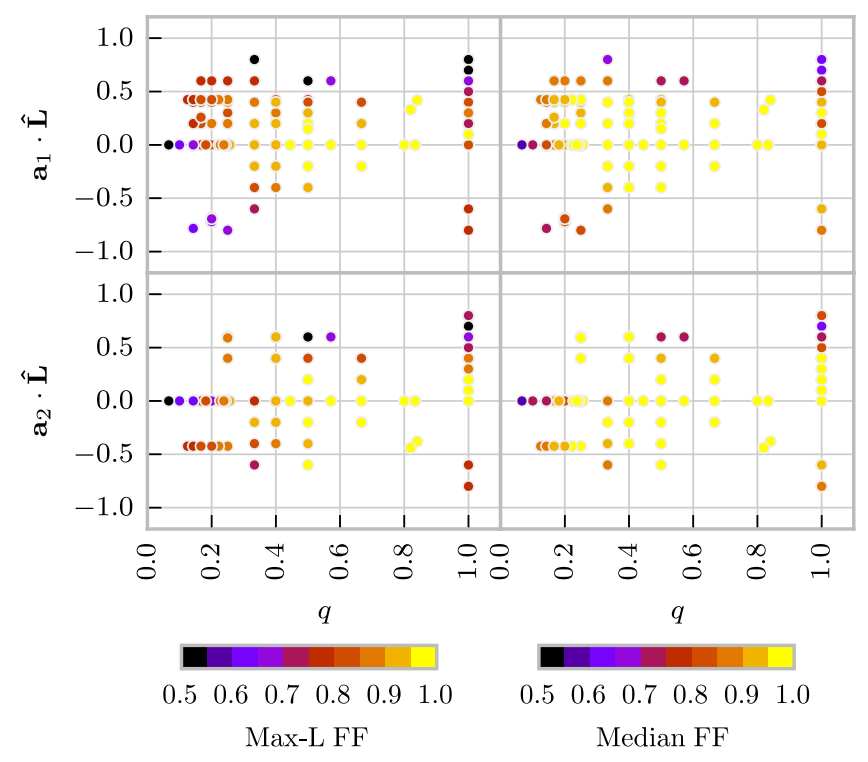

FIG. 12. Fitting factors between cWB (left) and BayesWave (right) and the NR waveforms, in terms of the mass ratio $q$ and the dot products between the component spins and the orbital angular momentum, $\mathbf{a}_{1} \cdot \hat{\mathbf{L}}, \mathbf{a}_{2} \cdot \hat{\mathbf{L}}$. The quoted BayesWave fitting factor values are the median values evaluated across 1000 posterior waveform samples. 
fitting factor across the spin space suggests this is not strongly constrained.

The BayesWave and cWB reconstructed waveforms have a fitting factor with the best fit NR waveform of 0.95 and 0.87 , respectively. Fits within $1 \%$ of the best fit value are achieved with detector frame total mass in the range 66.4-74.8 $\mathrm{M}_{\odot}$ for BayesWave and 67.9-75.7 $\mathrm{M}_{\odot}$ for $\mathrm{cWB}$. This is in excellent agreement with the range 66-75 $\mathrm{M}_{\odot}$ estimated using LALInference [29]. The chirp mass of NR waveforms within $1 \%$ of the best fit to the BayesWave and cWB reconstructions is in the range 27.4-32.6 $\mathrm{M}_{\odot}$ and 27.8-33.0 $\mathrm{M}_{\odot}$, again with close overlap to the LALInference result of $29-33 \mathrm{M}_{\odot}$.

In addition to matching parameter estimation performed using analytic waveform models in [29], the parameter bounds shown here are consistent with those obtained via the time-frequency analyses in Secs. VC and VD. Findings similar to those here are reported in [50] where a suite of NR waveforms, including those used in this study, are compared directly with the data in a novel Bayesian analysis. Again, the parameter space preferred by that study clearly overlaps with that here. The agreement between the analytic waveform results and the Bayesian NR analysis helps to validate the use of those waveform templates. Meanwhile, the overlap with the model-independent reconstructions here demonstrates that there is no significant additional signal content which the NR waveforms fail to represent, as would be the case for sources other than BBH. The concordance among the findings from these three studies further serves to highlight the $\mathrm{BBH}$ origin of GW150914.

\section{DISCUSSION}

All-sky searches for short-duration gravitationalwave bursts scan a broad parameter space to identify the presence of gravitational-wave signals in the data. They discovered GW150914 in a low-latency online analysis, and identified it as clearly distinct from detector noise events. Further analysis of GW150914 showed that the reconstructed waveform of the signal is consistent with expectations for a binary black hole merger. Outputs of the burst pipelines were also used to estimate the mass parameters of the source, in agreement with more specialized techniques.

The discovery of GW150914 is a turning point in gravitational-wave astronomy. At the time of the discovery, low-latency burst searches were configured to search a broad parameter space, similar to gravitational-wave burst searches performed during the initial detector era. The large search parameter space was seen to overlap with high-mass binary black hole signals in studies with simulated data, an observation confirmed by the detection of GW150914. Looking towards the future, the emphasis on searches with minimal assumptions of the waveform morphology allows for gravitational-wave burst searches to explore the vast discovery space of gravitational-wave transients from a variety of potential sources.

Beyond the challenge of detecting gravitational waves, burst parameter estimation tools, which make weak signal assumptions, have demonstrated their ability to extract astrophysical information about the progenitor of GW150914. Rapid sky localization of transient sources will facilitate multimessenger astronomy and allow for improved characterization of gravitational-wave signal progenitors. Many of the tools used for GW150914, such as waveform reconstruction, have applications beyond gravitational waves from binary coalescences.

The methods described in this work will also be used to search the full data set from the first observing run in the advanced detector era and beyond. Gravitational-wave burst searches have shown, through their detection and analysis of GW150914, that they are ready to contribute to an era of gravitational-wave astronomy.

\section{ACKNOWLEDGMENTS}

The authors gratefully acknowledge the support of the United States National Science Foundation (NSF) for the construction and operation of the LIGO Laboratory and Advanced LIGO as well as the Science and Technology Facilities Council (STFC) of the United Kingdom, the MaxPlanck-Society (MPS), and the State of Niedersachsen/ Germany for support of the construction of Advanced LIGO and construction and operation of the GEO 600 detector. Additional support for Advanced LIGO was provided by the Australian Research Council. The authors gratefully acknowledge the Italian Istituto Nazionale di Fisica Nucleare (INFN), the French Centre National de la Recherche Scientifique (CNRS) and the Foundation for Fundamental Research on Matter supported by the Netherlands Organisation for Scientific Research, for the construction and operation of the Virgo detector and the creation and support of the EGO consortium. The authors also gratefully acknowledge research support from these agencies as well as by the Council of Scientific and Industrial Research of India, Department of Science and Technology, India; Science \& Engineering Research Board (SERB), India; Ministry of Human Resource Development, India; the Spanish Ministerio de Economía y Competitividad; the Conselleria d'Economia i Competitivitat and Conselleria d'Educació; Cultura i Universitats of the Govern de les Illes Balears; the National Science Centre of Poland; the European Commission; the Royal Society; the Scottish Funding Council; the Scottish Universities Physics Alliance; the Hungarian Scientific Research Fund (OTKA); the Lyon Institute of Origins (LIO); the National Research Foundation of Korea; Industry Canada and the Province of Ontario through the Ministry of Economic Development and Innovation; the National Science and Engineering Research Council Canada; Canadian Institute for Advanced Research; the Brazilian Ministry of Science, Technology, and 
Innovation; Russian Foundation for Basic Research; the Leverhulme Trust; the Research Corporation; Ministry of Science and Technology (MOST), Taiwan; and the Kavli Foundation. The authors gratefully acknowledge the support of the NSF, STFC, MPS, INFN, CNRS and the State of Niedersachsen/Germany for provision of computational resources. This article has been assigned the document number LIGO-P1500229.
[1] J. Aasi et al., Advanced LIGO, Classical Quantum Gravity 32, 074001 (2015).

[2] B. P. Abbott et al., GW150914: The Advanced LIGO Detectors in the Era of First Discoveries, Phys. Rev. Lett. 116, 131103 (2016).

[3] A. Einstein, Näherungsweise integration der feldgleichungen der gravitation, Sitzungsberichte der Königlich Preußischen Akademie der Wissenschaften (Berlin) 1, 688 (1916).

[4] A. Einstein, Über gravitationswellen, Sitzungsberichte der Königlich Preußischen Akademie der Wissenschaften (Berlin), 1, 154 (1918).

[5] H. Luck et al., The upgrade of GEO600, J. Phys. Conf. Ser. 228, 012012 (2010).

[6] F. Acernese et al., Advanced Virgo: A second-generation interferometric gravitational wave detector, Classical Quantum Gravity 32, 024001 (2015).

[7] Y. Aso, Y. Michimura, K. Somiya, M. Ando, O. Miyakawa, T. Sekiguchi, D. Tatsumi, and H. Yamamoto, Interferometer design of the KAGRA gravitational wave detector, Phys. Rev. D 88, 043007 (2013).

[8] B. P. Abbott et al., Prospects for observing and localizing gravitational-wave transients with Advanced LIGO and Advanced Virgo, Living Rev. Relativ. 19, 1 (2016).

[9] N. Andersson et al., The transient gravitational-wave sky, Classical Quantum Gravity 30, 193002 (2013).

[10] C.L. Fryer and K. C. B. New, Gravitational waves from gravitational collapse, Living Rev. Relativity 14, 1 (2011).

[11] T. Damour and A. Vilenkin, Gravitational radiation from cosmic (super)strings: Bursts, stochastic background, and observational windows, Phys. Rev. D 71, 063510 (2005).

[12] K. S. Thorne, in 300 Years of Gravitation, edited by S. W. Hawking and W. Israel (Cambridge University Press, Cambridge, England, 1987).

[13] R. W. P. Drever, F. J. Raab, K. S. Thorne, R. Vogt, and R. Weiss, A laser interferometer gravitational-wave observatory (LIGO), Technical report, 1989, https://dcc.ligo.org/ LIGO-M890001/public/main.

[14] E. E. Flanagan and S. A. Hughes, Measuring gravitational waves from binary black hole coalescences: 2 . The waves' information and its extraction, with and without templates, Phys. Rev. D 57, 4566 (1998).

[15] J. Aasi et al., Search for gravitational radiation from intermediate mass black hole binaries in data from the second LIGO-Virgo joint science run, Phys. Rev. D 89, 122003 (2014).

[16] S. Mohapatra, L. Cadonati, S. Caudill, J. Clark, C. Hanna, S. Klimenko, C. Pankow, R. Vaulin, G. Vedovato, and S. Vitale, Sensitivity comparison of searches for binary black hole coalescences with ground-based gravitationalwave detectors, Phys. Rev. D 90, 022001 (2014).

[17] J. Abadie et al., All-sky search for gravitational-wave bursts in the second joint LIGO-Virgo run, Phys. Rev. D 85, 122007 (2012).

[18] B. P. Abbott et al., An all-sky search for long-duration gravitational wave transients with LIGO, Phys. Rev. D 93, 042005 (2016).

[19] J. Abadie et al., All-sky search for gravitational-wave bursts in the first joint LIGO-GEO-Virgo run, Phys. Rev. D 81, 102001 (2010).

[20] E. Thrane and M. Coughlin, Detecting Gravitation-Wave Transients at 5 Sigma: A Hierarchical Approach, Phys. Rev. Lett. 115, 181102 (2015).

[21] J. B. Kanner, T. B. Littenberg, N. Cornish, M. Millhouse, E. Xhakaj, F. Salemi, M. Drago, G. Vedovato, and S. Klimenko, Leveraging waveform complexity for confident detection of gravitational waves, Phys. Rev. D 93, 022002 (2016).

[22] T. B. Littenberg, J. B. Kanner, N. J. Cornish, and M. Millhouse, Enabling high confidence detections of gravitational-wave bursts, arXiv:1511.08752.

[23] R. Lynch, S. Vitale, R. Essick, E. Katsavounidis, and F. Robinet, An information-theoretic approach to the gravitational-wave burst detection problem, arXiv:1511.05955.

[24] S. Klimenko, G. Vedovato, M. Drago, F. Salemi, V. Tiwari, G. A. Prodi, C. Lazzaro, S. Tiwari, F. Da Silva, and G. Mitselmakher, Method for detection and reconstruction of gravitational wave transients with networks of advanced detectors, Phys. Rev. D 93, 042004 (2016).

[25] S. Klimenko, I. Yakushin, A. Mercer, and G. Mitselmakher, Coherent method for detection of gravitational wave bursts, Classical Quantum Gravity 25, 114029 (2008).

[26] B. P. Abbott et al., Observation of gravitational waves from a binary black hole merger, Phys. Rev. Lett. 116, 061102 (2016).

[27] B. P. Abbott et al., Characterization of transient noise in Advanced LIGO relevant to gravitational wave signal GW150914, arXiv:1602.03844.

[28] B. Abbott et al., Localization and broadband follow-up of the gravitational-wave transient GW150914, arXiv: 1602.08492 .

[29] B. P. Abbott et al., Properties of the binary black hole merger GW150914, arXiv:1602.03840.

[30] B. P. Abbott et al., GW150914: First results from the search for binary black hole coalescence with Advanced LIGO, arXiv:1602.03839.

[31] N. J. Cornish and T. B. Littenberg, BayesWave: Bayesian inference for gravitational wave bursts and instrument glitches, Classical Quantum Gravity 32, 135012 (2015). 
[32] W. G. Anderson, P. R. Brady, J. D. E. Creighton, and E. E. Flanagan, An excess power statistic for detection of burst sources of gravitational radiation, Phys. Rev. D 63, 042003 (2001).

[33] J. Aasi et al., Search for gravitational radiation from intermediate mass black hole binaries in data from the second LIGO-Virgo joint science run, Phys. Rev. D 89, 122003 (2014).

[34] V. Necula, S. Klimenko, and G. Mitselmakher, Transient analysis with fast Wilson-Daubechies time-frequency transform, J. Phys. Conf. Ser. 363, 012032 (2012).

[35] S. Klimenko, S. Mohanty, M. Rakhmanov, and G. Mitselmakher, Constraint likelihood analysis for a network of gravitational wave detectors, Phys. Rev. D 72, 122002 (2005).

[36] P. C. Peters, Gravitational radiation and the motion of two point masses, Phys. Rev. 136, B1224 (1964).

[37] S. Chatterji, L. Blackburn, G. Martin, and E. Katsavounidis, Multiresolution techniques for the detection of gravitational-wave bursts, Classical Quantum Gravity 21, S1809 (2004).

[38] F. Robinet, Omicron: An algorithm to detect and characterize transient noise in gravitational-wave detectors, https:// tds.ego-gw.it/q1/?c=10651, 2015.

[39] J. Skilling, Nested sampling for general Bayesian computation, Bayesian Anal. 1, 833 (2006).

[40] J. Veitch et al., Parameter estimation for compact binaries with ground-based gravitational-wave observations using the LALInference software library, Phys. Rev. D 91, 042003 (2015).

[41] T. B. Littenberg and N. J. Cornish, Bayesian inference for spectral estimation of gravitational wave detector noise, Phys. Rev. D 91, 084034 (2015).

[42] A. Taracchini et al., Effective-one-body model for blackhole binaries with generic mass ratios and spins, Phys. Rev. D 89, 061502 (2014).

[43] P. Kumar, K. Barkett, S. Bhagwat, N. Afshari, D. A. Brown, G. Lovelace, M. A. Scheel, and B. Szilágyi, Accuracy and precision of gravitational-wave models of inspiraling neutron star-black hole binaries with spin: Comparison with matter-free numerical relativity in the low-frequency regime, Phys. Rev. D 92, 102001 (2015).

[44] R. Essick, S. Vitale, E. Katsavounidis, G. Vedovato, and S. Klimenko, Localization of short duration gravitational-wave transients with the early advanced LIGO and Virgo detectors, Astrophys. J. 800, 81 (2015).

[45] B. P. Abbott et al., Calibration of the Advanced LIGO detectors for the discovery of the binary black-hole merger GW150914, arXiv:1602.03845.

[46] V. Tiwari, S. Klimenko, V. Necula, and G. Mitselmakher, Reconstruction of chirp mass in searches for gravitational wave transients, Classical Quantum Gravity 33, 01LT01 (2016).

[47] B. P. Abbott et al., Tests of general relativity with GW150914, arXiv:1602.03841.

[48] L. Pekowsky, R. O'Shaughnessy, J. Healy, and D. Shoemaker, Comparing gravitational waves from nonprecessing and precessing black hole binaries in the corotating frame, Phys. Rev. D 88, 024040 (2013).

[49] I. Hinder, A. Buonanno, M. Boyle, Z. B. Etienne, and J. Healy (NRAR Collaboration), Error-analysis and comparison to analytical models of numerical waveforms produced by the NRAR Collaboration, Classical Quantum Gravity 31, 025012 (2014).

[50] B. Abbott et al., Directly comparing GW150914 with numerical relativity (to be published).

[51] B. Allen, W. G. Anderson, P. R. Brady, D. A. Brown, and J. D. E. Creighton, FINDCHIRP: An algorithm for detection of gravitational waves from inspiraling compact binaries, Phys. Rev. D 85, 122006 (2012).

[52] J. A. Nelder and R. Mead, A simplex method for function minimization, Computer Journal (UK) 7, 308 (1965).

[53] S. Fischetti, J. Healy, L. Cadonati, L. London, S. R. P. Mohapatra, and D. Shoemaker, Exploring the use of numerical relativity waveforms in burst analysis of precessing black hole mergers, Phys. Rev. D 83, 044019 (2011).

[54] R. O'Shaughnessy, B. Vaishnav, J. Healy, and D. Shoemaker, Intrinsic selection biases of ground-based gravitational wave searches for high-mass BH-BH mergers, Phys. Rev. D 82, 104006 (2010).

[55] R. O'Shaughnessy, L. London, J. Healy, and D. Shoemaker, Precession during merger: Strong polarization changes are observationally accessible features of strong-field gravity during binary black hole merger, Phys. Rev. D 87, 044038 (2013).

[56] L. Pekowsky, J. Healy, D. Shoemaker, and P. Laguna, Impact of higher-order modes on the detection of binary black hole coalescences, Phys. Rev. D 87, 084008 (2013).

[57] J. Healy, P. Laguna, L. Pekowsky, and D. Shoemaker, Template mode hierarchies for binary black hole mergers, Phys. Rev. D 88, 024034 (2013).

[58] L. London, D. Shoemaker, and J. Healy, Modeling ringdown: Beyond the fundamental quasinormal modes, Phys. Rev. D 90, 124032 (2014).

[59] K. Jani, J. Healy, J. Clark, L. London, P. Laguna, and D. Shoemaker, Georgia tech catalog of gravitational waveforms (to be published).

[60] C. R. Galley and P. Schmidt, Fast and efficient evaluation of gravitational waveforms via reduced-order spline interpolation (to be published).

[61] P. Schmidt, I. W. Harry, and H. P. Pfeiffer, Numerical relativity injection infrastructure, LIGO Technical Document, https://dcc.ligo.org/LIGO-T1500606/public.

B. P. Abbott, ${ }^{1}$ R. Abbott, ${ }^{1}$ T. D. Abbott,${ }^{2}$ M. R. Abernathy, ${ }^{1}$ F. Acernese,${ }^{3,4}$ K. Ackley,${ }^{5}$ C. Adams,${ }^{6}$ T. Adams, ${ }^{7}$ P. Addesso, ${ }^{3}$ R. X. Adhikari, ${ }^{1}$ V. B. Adya,${ }^{8}$ C. Affeldt, ${ }^{8}$ M. Agathos, ${ }^{9}$ K. Agatsuma, ${ }^{9}$ N. Aggarwal, ${ }^{10}$ O. D. Aguiar, ${ }^{11}$ L. Aiello, ${ }^{12,13}$ A. Ain, ${ }^{14}$ P. Ajith, ${ }^{15}$ B. Allen,${ }^{8,16,17}$ A. Allocca, ${ }^{18,19}$ P. A. Altin ${ }^{20}$ S. B. Anderson, ${ }^{1}$ W. G. Anderson, ${ }^{16}$ K. Arai, ${ }^{1}$ M. C. Araya, ${ }^{1}$ C. C. Arceneaux ${ }^{21}$ J. S. Areeda, ${ }^{22}$ N. Arnaud,${ }^{23}$ K. G. Arun, ${ }^{24}$ S. Ascenzi, ${ }^{25,13}$ G. Ashton, ${ }^{26}$ M. Ast, ${ }^{27}$ S. M. Aston, ${ }^{6}$ 
P. Astone, ${ }^{28}$ P. Aufmuth, ${ }^{8}$ C. Aulbert,${ }^{8}$ S. Babak, ${ }^{29}$ P. Bacon,${ }^{30}$ M. K. M. Bader, ${ }^{9}$ P. T. Baker, ${ }^{31}$ F. Baldaccini, ${ }^{32,33}$ G. Ballardin, ${ }^{34}$ S. W. Ballmer, ${ }^{35}$ J. C. Barayoga, ${ }^{1}$ S. E. Barclay, ${ }^{36}$ B. C. Barish, ${ }^{1}$ D. Barker ${ }^{37}$ F. Barone, ${ }^{3,4}$ B. Barr, ${ }^{36}$ L. Barsotti, ${ }^{10}$ M. Barsuglia, ${ }^{30}$ D. Barta, ${ }^{38}$ J. Bartlett, ${ }^{37}$ I. Bartos, ${ }^{39}$ R. Bassiri, ${ }^{40}$ A. Basti, ${ }^{18,19}$ J. C. Batch, ${ }^{37}$ C. Baune, ${ }^{8}$ V. Bavigadda, ${ }^{34}$ M. Bazzan, ${ }^{41,42}$ B. Behnke, ${ }^{29}$ M. Bejger, ${ }^{43}$ A. S. Bell, ${ }^{36}$ C. J. Bell, ${ }^{36}$ B. K. Berger, ${ }^{1}$ J. Bergman, ${ }^{37}$ G. Bergmann, ${ }^{8}$ C. P. L. Berry, ${ }^{44}$ D. Bersanetti, ${ }^{45,46}$ A. Bertolini, ${ }^{9}$ J. Betzwieser, ${ }^{6}$ S. Bhagwat, ${ }^{35}$ R. Bhandare, ${ }^{47}$ I. A. Bilenko, ${ }^{48}$ G. Billingsley, ${ }^{1}$ J. Birch, ${ }^{6}$ R. Birney, ${ }^{49}$ S. Biscans, ${ }^{10}$ A. Bisht,${ }^{8,17}$ M. Bitossi, ${ }^{34}$ C. Biwer, ${ }^{35}$ M. A. Bizouard,${ }^{23}$ J. K. Blackburn, ${ }^{1}$ L. Blackburn, ${ }^{10}$ C. D. Blair, ${ }^{50}$ D. G. Blair, ${ }^{50}$ R. M. Blair, ${ }^{37}$ S. Bloemen,${ }^{51}$ O. Bock, ${ }^{8}$ T. P. Bodiya, ${ }^{10}$ M. Boer, ${ }^{52}$ G. Bogaert, ${ }^{52}$ C. Bogan, ${ }^{8}$ A. Bohe, ${ }^{29}$ P. Bojtos ${ }^{53}$ C. Bond,${ }^{44}$ F. Bondu, ${ }^{54}$ R. Bonnand, ${ }^{7}$ B. A. Boom, ${ }^{9}$ R. Bork, ${ }^{1}$

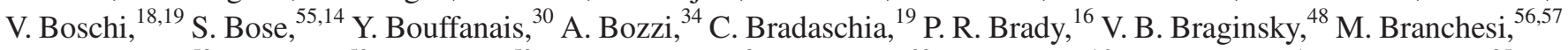
J. E. Brau, ${ }^{58}$ T. Briant, ${ }^{59}$ A. Brillet, ${ }^{52}$ M. Brinkmann, ${ }^{8}$ V. Brisson, ${ }^{23}$ P. Brockill, ${ }^{16}$ A. F. Brooks, ${ }^{1}$ D. A. Brown, ${ }^{35}$ D. D. Brown, ${ }^{44}$ N. M. Brown, ${ }^{10}$ C. C. Buchanan, ${ }^{2}$ A. Buikema, ${ }^{10}$ T. Bulik, ${ }^{60}$ H. J. Bulten, ${ }^{61,9}$ A. Buonanno, ${ }^{29,62}$ D. Buskulic, ${ }^{7}$ C. Buy, ${ }^{30}$ R. L. Byer, ${ }^{40}$ L. Cadonati, ${ }^{63}$ G. Cagnoli, ${ }^{64,65}$ C. Cahillane, ${ }^{1}$ J. Calderón Bustillo, ${ }^{66,63}$ T. Callister, ${ }^{1}$ E. Calloni, ${ }^{67,4}$ J. B. Camp, ${ }^{68}$ K. C. Cannon ${ }^{69}$ J. Cao,${ }^{70}$ C. D. Capano, ${ }^{8}$ E. Capocasa,${ }^{30}$ F. Carbognani,${ }^{34}$ S. Caride, ${ }^{71}$ J. Casanueva Diaz, ${ }^{23}$ C. Casentini, ${ }^{25,13}$ S. Caudill, ${ }^{16}$ M. Cavaglià, ${ }^{21}$ F. Cavalier,${ }^{23}$ R. Cavalieri, ${ }^{34}$ G. Cella, ${ }^{19}$ C. B. Cepeda, ${ }^{1}$ L. Cerboni Baiardi,${ }^{56,57}$ G. Cerretani, ${ }^{18,19}$ E. Cesarini ${ }^{25,13}$ R. Chakraborty, ${ }^{1}$ S. Chatterji, ${ }^{10}$ T. Chalermsongsak, ${ }^{1}$ S. J. Chamberlin, ${ }^{72}$ M. Chan, ${ }^{36}$ S. Chao,${ }^{73}$ P. Charlton, ${ }^{74}$ E. Chassande-Mottin, ${ }^{30}$ H. Y. Chen, ${ }^{75}$ Y. Chen, ${ }^{76}$ C. Cheng, ${ }^{73}$ A. Chincarini, ${ }^{46}$ A. Chiummo, ${ }^{34}$ H. S. Cho, ${ }^{77}$ M. Cho, ${ }^{62}$ J. H. Chow, ${ }^{20}$ N. Christensen, ${ }^{78}$ Q. Chu, ${ }^{50}$ S. Chua, ${ }^{59}$ S. Chung,${ }^{50}$ G. Ciani, ${ }^{5}$ F. Clara, ${ }^{37}$ J. A. Clark, ${ }^{63}$ M. Clark, ${ }^{63}$ F. Cleva, ${ }^{52}$ E. Coccia, ${ }^{25,12,13}$ P.-F. Cohadon, ${ }^{59}$ A. Colla, ${ }^{79,28}$ C. G. Collette, ${ }^{80}$ L. Cominsky, ${ }^{81}$ M. Constancio Jr., ${ }^{11}$ A. Conte, ${ }^{79,28}$ L. Conti, ${ }^{42}$ D. Cook,${ }^{37}$ T. R. Corbitt, ${ }^{2}$ N. Cornish, ${ }^{31}$ A. Corsi, ${ }^{71}$

S. Cortese, ${ }^{34}$ C. A. Costa, ${ }^{11}$ M. W. Coughlin, ${ }^{78}$ S. B. Coughlin, ${ }^{82}$ J.-P. Coulon, ${ }^{52}$ S. T. Countryman, ${ }^{39}$ P. Couvares, ${ }^{1}$ E. E. Cowan, ${ }^{63}$ D. M. Coward, ${ }^{50}$ M. J. Cowart, ${ }^{6}$ D. C. Coyne, ${ }^{1}$ R. Coyne, ${ }^{71}$ K. Craig, ${ }^{36}$ J. D. E. Creighton, ${ }^{16}$ J. Cripe, ${ }^{2}$ S. G. Crowder, ${ }^{83}$ A. Cumming, ${ }^{36}$ L. Cunningham, ${ }^{36}$ E. Cuoco, ${ }^{34}$ T. Dal Canton, ${ }^{8}$ S. L. Danilishin, ${ }^{36}$ S. D'Antonio, ${ }^{13}$ K. Danzmann, ${ }^{17,8}$ N. S. Darman, ${ }^{84}$ V. Dattilo, ${ }^{34}$ I. Dave, ${ }^{47}$ H. P. Daveloza,${ }^{85}$ M. Davier, ${ }^{23}$ G. S. Davies, ${ }^{36}$ E. J. Daw, ${ }^{86}$ R. Day, ${ }^{34}$ D. DeBra, ${ }^{40}$ G. Debreczeni, ${ }^{38}$ J. Degallaix, ${ }^{65}$ M. De Laurentis,${ }^{67,4}$ S. Deléglise, ${ }^{59}$ W. Del Pozzo ${ }^{44}$ T. Denker, ${ }^{8,17}$ T. Dent, ${ }^{8}$ H. Dereli, ${ }^{52}$ V. Dergachev, ${ }^{1}$ R. T. DeRosa, ${ }^{6}$ R. De Rosa,${ }^{67,4}$ R. DeSalvo, ${ }^{87}$ S. Dhurandhar, ${ }^{14}$ M. C. Díaz, ${ }^{85}$ L. Di Fiore, ${ }^{4}$ M. Di Giovanni, ${ }^{79,28}$ A. Di Lieto, ${ }^{18,19}$ S. Di Pace ${ }^{79,28}$ I. Di Palma, ${ }^{29,8}$ A. Di Virgilio, ${ }^{19}$ G. Dojcinoski, ${ }^{88}$ V. Dolique, ${ }^{65}$ F. Donovan, ${ }^{10}$ K. L. Dooley, ${ }^{21}$ S. Doravari, ${ }^{6,8}$ R. Douglas, ${ }^{36}$ T. P. Downes,${ }^{16}$ M. Drago, ${ }^{8,89,90}$ R. W. P. Drever, ${ }^{1}$ J. C. Driggers, ${ }^{37}$ Z. Du, ${ }^{70}$ M. Ducrot, ${ }^{7}$ S. E. Dwyer, ${ }^{37}$ T. B. Edo, ${ }^{86}$ M. C. Edwards, ${ }^{78}$ A. Effler, ${ }^{6}$ H.-B. Eggenstein, ${ }^{8}$ P. Ehrens, ${ }^{1}$ J. Eichholz, ${ }^{5}$ S. S. Eikenberry, ${ }^{5}$ W. Engels, ${ }^{76}$ R. C. Essick, ${ }^{10}$ T. Etzel, ${ }^{1}$ M. Evans, ${ }^{10}$ T. M. Evans, ${ }^{6}$ R. Everett, ${ }^{72}$ M. Factourovich, ${ }^{39}$ V. Fafone, ${ }^{25,13,12}$ H. Fair, ${ }^{35}$ S. Fairhurst, ${ }^{91}$ X. Fan, ${ }^{70}$ Q. Fang, ${ }^{50}$ S. Farinon, ${ }^{46}$ B. Farr, ${ }^{75}$ W. M. Farr, ${ }^{44}$ M. Favata ${ }^{88}$ M. Fays, ${ }^{91}$ H. Fehrmann, ${ }^{8}$ M. M. Fejer, ${ }^{40}$ I. Ferrante, ${ }^{18,19}$ E. C. Ferreira, ${ }^{11}$ F. Ferrini, ${ }^{34}$ F. Fidecaro, ${ }^{18,19}$ I. Fiori, ${ }^{34}$ D. Fiorucci, ${ }^{30}$ R. P. Fisher, ${ }^{35}$ R. Flaminio, ${ }^{65,92}$ M. Fletcher, ${ }^{36}$ J.-D. Fournier, ${ }^{52}$ S. Franco, ${ }^{23}$ S. Frasca, ${ }^{79,28}$ F. Frasconi, ${ }^{19}$ Z. Frei, ${ }^{53}$ A. Freise, ${ }^{44}$ R. Frey, ${ }^{58}$ V. Frey, ${ }^{23}$ T. T. Fricke, ${ }^{8}$ P. Fritschel,${ }^{10}$ V. V. Frolov, ${ }^{6}$ P. Fulda, ${ }^{5}$ M. Fyffe, ${ }^{6}$

H. A. G. Gabbard, ${ }^{21}$ J. R. Gair, ${ }^{93}$ L. Gammaitoni, ${ }^{32,33}$ S. G. Gaonkar, ${ }^{14}$ F. Garufi,,${ }^{67,4}$ A. Gatto, ${ }^{30}$ G. Gaur, ${ }^{94,95}$ N. Gehrels, ${ }^{68}$ G. Gemme, ${ }^{46}$ B. Gendre, ${ }^{52}$ E. Genin, ${ }^{34}$ A. Gennai, ${ }^{19}$ J. George,${ }^{47}$ L. Gergely, ${ }^{96}$ V. Germain, ${ }^{7}$ Archisman Ghosh, ${ }^{15}$ S. Ghosh, ${ }^{51,9}$ J. A. Giaime, ${ }^{2,6}$ K. D. Giardina, ${ }^{6}$ A. Giazotto, ${ }^{19}$ K. Gill, ${ }^{97}$ A. Glaefke, ${ }^{36}$ E. Goetz, ${ }^{98}$ R. Goetz,${ }^{5}$ L. Gondan, ${ }^{53}$ G. González, ${ }^{2}$ J. M. Gonzalez Castro, ${ }^{18,19}$ A. Gopakumar, ${ }^{99}$ N. A. Gordon, ${ }^{36}$ M. L. Gorodetsky, ${ }^{48}$ S. E. Gossan, ${ }^{1}$ M. Gosselin, ${ }^{34}$ R. Gouaty, ${ }^{7}$ C. Graef,${ }^{36}$ P. B. Graff, ${ }^{62}$ M. Granata ${ }^{65}$ A. Grant,${ }^{36}$ S. Gras, ${ }^{10}$ C. Gray, ${ }^{37}$ G. Greco, ${ }^{56,57}$ A. C. Green, ${ }^{44}$ P. Groot, ${ }^{51}$ H. Grote, ${ }^{8}$ S. Grunewald, ${ }^{29}$ G. M. Guidi, ${ }^{56,57}$ X. Guo, ${ }^{70}$ A. Gupta, ${ }^{14}$ M. K. Gupta, ${ }^{95}$ K. E. Gushwa, ${ }^{1}$ E. K. Gustafson, ${ }^{1}$ R. Gustafson, ${ }^{98}$ R. Haas, ${ }^{29}$ J. J. Hacker, ${ }^{22}$ B. R. Hall, ${ }^{55}$ E. D. Hall, ${ }^{1}$ G. Hammond ${ }^{36}$ M. Haney, ${ }^{99}$ M. M. Hanke, ${ }^{8}$ J. Hanks, ${ }^{37}$ C. Hanna, ${ }^{72}$ M. D. Hannam, ${ }^{91}$ J. Hanson, ${ }^{6}$ T. Hardwick, ${ }^{2}$ J. Harms,${ }^{56,57}$ G. M. Harry, ${ }^{100}$ I. W. Harry, ${ }^{29}$ M. J. Hart, ${ }^{36}$ M. T. Hartman, ${ }^{5}$ C.-J. Haster, ${ }^{44}$ K. Haughian, ${ }^{36}$ J. Healy, ${ }^{112}$ A. Heidmann, ${ }^{59}$ M. C. Heintze, ${ }^{5,6}$ H. Heitmann, ${ }^{52}$ P. Hello, ${ }^{23}$ G. Hemming, ${ }^{34}$ M. Hendry, ${ }^{36}$ I. S. Heng, ${ }^{36}$ J. Hennig, ${ }^{36}$ A. W. Heptonstall, ${ }^{1}$ M. Heurs, ${ }^{8,17}$ S. Hild, ${ }^{36}$ I. Hinder, ${ }^{29}$ D. Hoak, ${ }^{101}$ K. A. Hodge, ${ }^{1}$ D. Hofman, ${ }^{65}$ S. E. Hollitt, ${ }^{102}$ K. Holt, ${ }^{6}$ D. E. Holz, ${ }^{75}$ P. Hopkins, ${ }^{91}$ D. J. Hosken, ${ }^{102}$ J. Hough, ${ }^{36}$ E. A. Houston, ${ }^{36}$ E. J. Howell, ${ }^{50}$ Y. M. Hu, ${ }^{36}$ S. Huang, ${ }^{73}$ E. A. Huerta, ${ }^{103,82}$ D. Huet, ${ }^{23}$ B. Hughey, ${ }^{97}$ S. Husa, ${ }^{66}$ S. H. Huttner, ${ }^{36}$ T. Huynh-Dinh, ${ }^{6}$ A. Idrisy, ${ }^{72}$ N. Indik, ${ }^{8}$ D. R. Ingram, ${ }^{37}$ R. Inta, ${ }^{71}$ H. N. Isa, ${ }^{36}$ J.-M. Isac, ${ }^{59}$ M. Isi, ${ }^{1}$ G. Islas, ${ }^{22}$ T. Isogai,${ }^{10}$ B. R. Iyer, ${ }^{15}$ K. Izumi, ${ }^{37}$ T. Jacqmin, ${ }^{59}$ H. Jang, ${ }^{77}$ K. Jani, ${ }^{63}$ P. Jaranowski, ${ }^{104}$ S. Jawahar, ${ }^{105}$ F. Jiménez-Forteza, ${ }^{66}$ W. W. Johnson, ${ }^{2}$ D. I. Jones,${ }^{26}$ R. Jones, ${ }^{36}$ R. J. G. Jonker, ${ }^{9}$ L. Ju, ${ }^{50}$ Haris K., ${ }^{106}$ C. V. Kalaghatgi, ${ }^{24,91}$ V. Kalogera, ${ }^{82}$ S. Kandhasamy, ${ }^{21}$ G. Kang, ${ }^{77}$ J. B. Kanner, ${ }^{1}$ S. Karki, ${ }^{58}$ 
M. Kasprzack,${ }^{2,23,34}$ E. Katsavounidis, ${ }^{10}$ W. Katzman, ${ }^{6}$ S. Kaufer,${ }^{17}$ T. Kaur, ${ }^{50}$ K. Kawabe, ${ }^{37}$ F. Kawazoe,${ }^{8,17}$ F. Kéfélian, ${ }^{52}$ M. S. Kehl ${ }^{69}$ D. Keitel, ${ }^{8,66}$ D. B. Kelley, ${ }^{35}$ W. Kells, ${ }^{1}$ R. Kennedy ${ }^{86}$ J. S. Key, ${ }^{85}$ A. Khalaidovski, ${ }^{8}$ F. Y. Khalili, ${ }^{48}$ I. Khan, ${ }^{12}$

S. Khan, ${ }^{91}$ Z. Khan, ${ }^{95}$ E. A. Khazanov, ${ }^{107}$ N. Kijbunchoo, ${ }^{37}$ C. Kim, ${ }^{77}$ J. Kim, ${ }^{108}$ K. Kim, ${ }^{109}$ Nam-Gyu Kim, ${ }^{77}$ Namjun Kim, ${ }^{40}$ Y.-M. Kim, ${ }^{108}$ E. J. King, ${ }^{102}$ P. J. King, ${ }^{37}$ M. Kinsey ${ }^{63}$ D. L. Kinzel, ${ }^{6}$ J. S. Kissel, ${ }^{37}$ L. Kleybolte, ${ }^{27}$ S. Klimenko, ${ }^{5}$ S. M. Koehlenbeck, ${ }^{8}$ K. Kokeyama, ${ }^{2}$ S. Koley, ${ }^{9}$ V. Kondrashov, ${ }^{1}$ A. Kontos, ${ }^{10}$ M. Korobko, ${ }^{27}$ W. Z. Korth, ${ }^{1}$ I. Kowalska, ${ }^{60}$ D. B. Kozak, ${ }^{1}$ V. Kringel, ${ }^{8}$ A. Królak, ${ }^{110,111}$ C. Krueger, ${ }^{17}$ G. Kuehn, ${ }^{8}$ P. Kumar, ${ }^{69}$ L. Kuo,${ }^{73}$ A. Kutynia, ${ }^{110}$ B. D. Lackey, ${ }^{35}$ P. Laguna, ${ }^{63}$ M. Landry, ${ }^{37}$ J. Lange, ${ }^{112}$ B. Lantz ${ }^{40}$ P. D. Lasky, ${ }^{113}$ A. Lazzarini, ${ }^{1}$ C. Lazzaro, ${ }^{63,42}$ P. Leaci, ${ }^{29,79,28}$ S. Leavey, ${ }^{36}$ E. O. Lebigot, ${ }^{30,70}$ C. H. Lee, ${ }^{108}$ H. K. Lee, ${ }^{109}$ H. M. Lee, ${ }^{114}$ K. Lee, ${ }^{36}$ A. Lenon, ${ }^{35}$ M. Leonardi, ${ }^{89,90}$ J. R. Leong, ${ }^{8}$ N. Leroy, ${ }^{23}$ N. Letendre, ${ }^{7}$ Y. Levin, ${ }^{113}$ B. M. Levine, ${ }^{37}$ T. G. F. Li, ${ }^{1}$ A. Libson, ${ }^{10}$ T. B. Littenberg, ${ }^{115}$ N. A. Lockerbie, ${ }^{105}$ J. Logue, ${ }^{36}$ A. L. Lombardi, ${ }^{101}$ J. E. Lord, ${ }^{35}$ M. Lorenzini, ${ }^{12,13}$ V. Loriette, ${ }^{116}$ M. Lormand, ${ }^{6}$ G. Losurdo, ${ }^{57}$ J. D. Lough, ${ }^{8,17}$ H. Lück, ${ }^{17,8}$ A. P. Lundgren, ${ }^{8}$ J. Luo, ${ }^{78}$ R. Lynch, ${ }^{10}$ Y. Ma,${ }^{50}$ T. MacDonald, ${ }^{40}$

B. Machenschalk, ${ }^{8}$ M. MacInnis, ${ }^{10}$ D. M. Macleod, ${ }^{2}$ F. Magaña-Sandoval,${ }^{35}$ R. M. Magee ${ }^{55}$ M. Mageswaran, ${ }^{1}$ E. Majorana ${ }^{28}$ I. Maksimovic, ${ }^{116}$ V. Malvezzi, ${ }^{25,13}$ N. Man ${ }^{52}$ I. Mandel,${ }^{44}$ V. Mandic, ${ }^{83}$ V. Mangano, ${ }^{36}$ G. L. Mansell, ${ }^{20}$ M. Manske, ${ }^{16}$ M. Mantovani, ${ }^{34}$ F. Marchesoni, ${ }^{117,33}$ F. Marion, ${ }^{7}$ S. Márka, ${ }^{39}$ Z. Márka, ${ }^{39}$ A. S. Markosyan, ${ }^{40}$ E. Maros, ${ }^{1}$ F. Martelli, ${ }^{56,57}$ L. Martellini, ${ }^{52}$ I. W. Martin ${ }^{36}$ R. M. Martin, ${ }^{5}$ D. V. Martynov, ${ }^{1}$ J. N. Marx, ${ }^{1}$ K. Mason, ${ }^{10}$ A. Masserot, ${ }^{7}$

T. J. Massinger, ${ }^{35}$ M. Masso-Reid,${ }^{36}$ F. Matichard ${ }^{10}$ L. Matone,${ }^{39}$ N. Mavalvala, ${ }^{10}$ N. Mazumder, ${ }^{55}$ G. Mazzolo, ${ }^{8}$

R. McCarthy, ${ }^{37}$ D. E. McClelland ${ }^{20}$ S. McCormick,${ }^{6}$ S. C. McGuire, ${ }^{118}$ G. McIntyre, ${ }^{1}$ J. McIver, ${ }^{1}$ D. J. McManus, ${ }^{20}$ S. T. McWilliams, ${ }^{103}$ D. Meacher, ${ }^{72}$ G. D. Meadors,${ }^{29,8}$ J. Meidam, ${ }^{9}$ A. Melatos ${ }^{84}$ G. Mendell, ${ }^{37}$ D. Mendoza-Gandara, ${ }^{8}$ R. A. Mercer, ${ }^{16}$ E. Merilh, ${ }^{37}$ M. Merzougui, ${ }^{52}$ S. Meshkov, ${ }^{1}$ C. Messenger,${ }^{36}$ C. Messick, ${ }^{72}$ P. M. Meyers, ${ }^{83}$ F. Mezzani,${ }^{28,79}$ H. Miao ${ }^{44}$ C. Michel,${ }^{65}$ H. Middleton, ${ }^{44}$ E. E. Mikhailov, ${ }^{119}$ L. Milano,${ }^{67,4}$ J. Miller, ${ }^{10}$ M. Millhouse, ${ }^{31}$ Y. Minenkov, ${ }^{13}$ J. Ming, ${ }^{29,8}$ S. Mirshekari, ${ }^{120}$ C. Mishra, ${ }^{15}$ S. Mitra, ${ }^{14}$ V. P. Mitrofanov, ${ }^{48}$ G. Mitselmakher, ${ }^{5}$ R. Mittleman, ${ }^{10}$ A. Moggi, ${ }^{19}$

M. Mohan, ${ }^{34}$ S. R. P. Mohapatra, ${ }^{10}$ M. Montani,${ }^{56,57}$ B. C. Moore,${ }^{88}$ C. J. Moore, ${ }^{121}$ D. Moraru,${ }^{37}$ G. Moreno, ${ }^{37}$

S. R. Morriss ${ }^{85}$ K. Mossavi, ${ }^{8}$ B. Mours, ${ }^{7}$ C. M. Mow-Lowry, ${ }^{44}$ C. L. Mueller, ${ }^{5}$ G. Mueller, ${ }^{5}$ A. W. Muir, ${ }^{91}$ Arunava Mukherjee, ${ }^{15}$ D. Mukherjee, ${ }^{16}$ S. Mukherjee, ${ }^{85}$ N. Mukund,${ }^{14}$ A. Mullavey, ${ }^{6}$ J. Munch, ${ }^{102}$ D. J. Murphy, ${ }^{39}$ P. G. Murray, ${ }^{36}$ A. Mytidis, ${ }^{5}$ I. Nardecchia, ${ }^{25,13}$ L. Naticchioni, ${ }^{79,28}$ R. K. Nayak, ${ }^{122}$ V. Necula, ${ }^{5}$ K. Nedkova, ${ }^{101}$ G. Nelemans, ${ }^{51,9}$ M. Neri, ${ }^{45,46}$ A. Neunzert, ${ }^{98}$ G. Newton, ${ }^{36}$ T. T. Nguyen, ${ }^{20}$ A. B. Nielsen, ${ }^{8}$ S. Nissanke,${ }^{51,9}$ A. Nitz, ${ }^{8}$ F. Nocera, ${ }^{34}$ D. Nolting, ${ }^{6}$ M. E. Normandin, ${ }^{85}$ L. K. Nuttall,${ }^{35}$ J. Oberling, ${ }^{37}$ E. Ochsner, ${ }^{16}$ J. O'Dell, ${ }^{123}$ E. Oelker, ${ }^{10}$ G. H. Ogin, ${ }^{124}$ J. J. Oh, ${ }^{125}$ S. H. Oh, ${ }^{125}$ F. Ohme, ${ }^{91}$ M. Oliver, ${ }^{66}$ P. Oppermann, ${ }^{8}$ Richard J. Oram, ${ }^{6}$ B. O'Reilly, ${ }^{6}$ R. O'Shaughnessy, ${ }^{12}$ C. D. Ott,${ }^{76}$ D. J. Ottaway, ${ }^{102}$ R. S. Ottens, ${ }^{5}$ H. Overmier, ${ }^{6}$ B. J. Owen, ${ }^{71}$ A. Pai, ${ }^{106}$ S. A. Pai ${ }^{47}$ J. R. Palamos, ${ }^{58}$ O. Palashov, ${ }^{107}$ C. Palomba, ${ }^{28}$ A. Pal-Singh,${ }^{27}$ H. Pan, ${ }^{73}$ C. Pankow, ${ }^{82}$ F. Pannarale, ${ }^{91}$ B. C. Pant, ${ }^{47}$ F. Paoletti, ${ }^{34,19}$ A. Paoli, ${ }^{34}$ M. A. Papa,${ }^{29,16,8}$ J. Page, ${ }^{115}$ H. R. Paris, ${ }^{40}$ W. Parker, ${ }^{6}$ D. Pascucci, ${ }^{36}$ A. Pasqualetti, ${ }^{34}$ R. Passaquieti, ${ }^{18,19}$ D. Passuello, ${ }^{19}$ B. Patricelli, ${ }^{18,19}$ Z. Patrick, ${ }^{40}$ B. L. Pearlstone, ${ }^{36}$ M. Pedraza, ${ }^{1}$ R. Pedurand,${ }^{65}$ L. Pekowsky, ${ }^{35}$ A. Pele, ${ }^{6}$ S. Penn, ${ }^{126}$ A. Perreca, ${ }^{1}$ M. Phelps,${ }^{36}$ O. Piccinni, ${ }^{79,28}$ M. Pichot, ${ }^{52}$ F. Piergiovanni, ${ }^{56,57}$ V. Pierro, ${ }^{87}$ G. Pillant ${ }^{34}$ L. Pinard, ${ }^{65}$ I. M. Pinto, ${ }^{87}$ M. Pitkin, ${ }^{36}$ R. Poggiani,,${ }^{18,19}$ P. Popolizio, ${ }^{34}$ A. Post,${ }^{8}$ J. Powell, ${ }^{36}$ J. Prasad,${ }^{14}$ V. Predoi, ${ }^{91}$ S. S. Premachandra, ${ }^{113}$ T. Prestegard ${ }^{83}$ L. R. Price, ${ }^{1}$ M. Prijatelj, ${ }^{34}$ M. Principe, ${ }^{87}$ S. Privitera,${ }^{29}$ G. A. Prodi,,${ }^{89,90}$ L. Prokhorov, ${ }^{48}$ O. Puncken, ${ }^{8}$ M. Punturo, ${ }^{33}$ P. Puppo, ${ }^{28}$ M. Pürrer, ${ }^{29}$ H. Qi,${ }^{16}$ J. Qin, ${ }^{50}$ V. Quetschke, ${ }^{85}$ E. A. Quintero, ${ }^{1}$ R. Quitzow-James, ${ }^{58}$ F. J. Raab, ${ }^{37}$ D. S. Rabeling,${ }^{20}$ H. Radkins, ${ }^{37}$ P. Raffai,${ }^{53}$ S. Raja, ${ }^{47}$ M. Rakhmanov, ${ }^{85}$ P. Rapagnani, ${ }^{79,28}$ V. Raymond, ${ }^{29}$ M. Razzano, ${ }^{18,19}$ V. Re, ${ }^{25}$ J. Read, ${ }^{22}$ C. M. Reed,${ }^{37}$ T. Regimbau, ${ }^{52}$ L. Rei, ${ }^{46}$ S. Reid, ${ }^{49}$ D. H. Reitze, ${ }^{1,5}$ H. Rew, ${ }^{119}$ S. D. Reyes, ${ }^{35}$ F. Ricci, ${ }^{79,28}$ K. Riles, ${ }^{98}$ N. A. Robertson, ${ }^{1,36}$ R. Robie, ${ }^{36}$ F. Robinet, ${ }^{23}$ A. Rocchi, ${ }^{13}$ L. Rolland, ${ }^{7}$ J. G. Rollins, ${ }^{1}$ V. J. Roma, ${ }^{58}$ R. Romano, ${ }^{3,4}$ G. Romanov, ${ }^{119}$ J. H. Romie, ${ }^{6}$ D. Rosińska, ${ }^{127,43}$ S. Rowan, ${ }^{36}$ A. Rüdiger, ${ }^{8}$

P. Ruggi, ${ }^{34}$ K. Ryan, ${ }^{37}$ S. Sachdev, ${ }^{1}$ T. Sadecki, ${ }^{37}$ L. Sadeghian, ${ }^{16}$ L. Salconi, ${ }^{34}$ M. Saleem, ${ }^{106}$ F. Salemi, ${ }^{8}$ A. Samajdar, ${ }^{122}$ L. Sammut, ${ }^{84,113}$ E. J. Sanchez, ${ }^{1}$ V. Sandberg, ${ }^{37}$ B. Sandeen, ${ }^{82}$ J. R. Sanders, ${ }^{98,35}$ B. Sassolas, ${ }^{65}$ B. S. Sathyaprakash, ${ }^{91}$ P. R. Saulson, ${ }^{35}$ O. Sauter, ${ }^{98}$ R. L. Savage, ${ }^{37}$ A. Sawadsky, ${ }^{17}$ P. Schale,${ }^{58}$ R. Schilling, ${ }^{8,}$ J. Schmidt, ${ }^{8}$ P. Schmidt, ${ }^{1,76}$

R. Schnabel, ${ }^{27}$ R. M. S. Schofield, ${ }^{58}$ A. Schönbeck, ${ }^{27}$ E. Schreiber, ${ }^{8}$ D. Schuette, ${ }^{8,17}$ B. F. Schutz, ${ }^{91,29}$ J. Scott, ${ }^{36}$ S. M. Scott, ${ }^{20}$ D. Sellers, ${ }^{6}$ A. S. Sengupta, ${ }^{94}$ D. Sentenac, ${ }^{34}$ V. Sequino, ${ }^{25,13}$ A. Sergeev, ${ }^{107}$ G. Serna, ${ }^{22}$ Y. Setyawati, ${ }^{51,9}$ A. Sevigny, ${ }^{37}$ D. A. Shaddock, ${ }^{20}$ S. Shah, ${ }^{51,9}$ M. S. Shahriar, ${ }^{82}$ M. Shaltev,${ }^{8}$ Z. Shao, ${ }^{1}$ B. Shapiro, ${ }^{40}$ P. Shawhan, ${ }^{62}$ A. Sheperd, ${ }^{16}$ D. H. Shoemaker, ${ }^{10}$ D. M. Shoemaker,${ }^{63}$ K. Siellez, ${ }^{52,63}$ X. Siemens, ${ }^{16}$ D. Sigg, ${ }^{37}$ A. D. Silva, ${ }^{11}$ D. Simakov, ${ }^{8}$ A. Singer, ${ }^{1}$ L. P. Singer, ${ }^{68}$ A. Singh ${ }^{29,8}$ R. Singh, ${ }^{2}$ A. Singhal, ${ }^{12}$ A. M. Sintes ${ }^{66}$ B. J. J. Slagmolen, ${ }^{20}$ J. R. Smith, ${ }^{22}$ N. D. Smith, ${ }^{1}$ R. J. E. Smith, ${ }^{1}$ E. J. Son, ${ }^{125}$ B. Sorazu, ${ }^{36}$ F. Sorrentino, ${ }^{46}$ T. Souradeep, ${ }^{14}$ A. K. Srivastava, ${ }^{95}$ A. Staley, ${ }^{39}$ 
M. Steinke, ${ }^{8}$ J. Steinlechner, ${ }^{36}$ S. Steinlechner, ${ }^{36}$ D. Steinmeyer, ${ }^{8,17}$ B. C. Stephens, ${ }^{16}$ R. Stone, ${ }^{85}$ K. A. Strain, ${ }^{36}$ N. Straniero, ${ }^{65}$ G. Stratta, ${ }^{56,57}$ N. A. Strauss, ${ }^{78}$ S. Strigin, ${ }^{48}$ R. Sturani, ${ }^{120}$ A. L. Stuver, ${ }^{6}$ T. Z. Summerscales, ${ }^{128}$ L. Sun, ${ }^{84}$ P. J. Sutton, ${ }^{91}$ B. L. Swinkels, ${ }^{34}$ M. J. Szczepańczyk, ${ }^{97}$ M. Tacca, ${ }^{30}$ D. Talukder,${ }^{58}$ D. B. Tanner, ${ }^{5}$ M. Tápai,${ }^{96}$ S. P. Tarabrin, ${ }^{8}$ A. Taracchini, ${ }^{29}$ R. Taylor, ${ }^{1}$ T. Theeg, ${ }^{8}$ M. P. Thirugnanasambandam, ${ }^{1}$ E. G. Thomas,${ }^{44}$ M. Thomas, ${ }^{6}$ P. Thomas, ${ }^{37}$ K. A. Thorne ${ }^{6}$ K. S. Thorne, ${ }^{76}$ E. Thrane, ${ }^{113}$ S. Tiwari, ${ }^{12}$ V. Tiwari, ${ }^{91}$ K. V. Tokmakov, ${ }^{105}$ C. Tomlinson, ${ }^{86}$ M. Tonelli, ${ }^{18,19}$ C. V. Torres, ${ }^{85, \dagger}$ C. I. Torrie, ${ }^{1}$ D. Töyrä, ${ }^{44}$ F. Travasso, ${ }^{32,33}$ G. Traylor, ${ }^{6}$ D. Trifirò, ${ }^{21}$ M. C. Tringali ${ }^{89,90}$ L. Trozzo, ${ }^{129,19}$ M. Tse, ${ }^{10}$ M. Turconi, ${ }^{52}$ D. Tuyenbayev,${ }^{85}$ D. Ugolini, ${ }^{130}$ C. S. Unnikrishnan, ${ }^{99}$ A. L. Urban, ${ }^{16}$ S. A. Usman, ${ }^{35}$ H. Vahlbruch, ${ }^{17}$ G. Vajente, ${ }^{1}$ G. Valdes, ${ }^{85}$ N. van Bakel,,${ }^{9}$ M. van Beuzekom,,${ }^{9}$ J. F. J. van den Brand,${ }^{61,9}$ C. Van Den Broeck, ${ }^{9}$ D. C. Vander-Hyde, ${ }^{35,22}$ L. van der Schaaf, ${ }^{9}$ J. V. van Heijningen, ${ }^{9}$ A. A. van Veggel, ${ }^{36}$ M. Vardaro, ${ }^{41,42}$ S. Vass, ${ }^{1}$ M. Vasúth, ${ }^{38}$ R. Vaulin, ${ }^{10}$ A. Vecchio, ${ }^{44}$ G. Vedovato, ${ }^{42}$ J. Veitch, ${ }^{44}$ P. J. Veitch, ${ }^{102}$ K. Venkateswara, ${ }^{131}$ D. Verkindt, ${ }^{7}$ F. Vetrano, ${ }^{56,57}$ A. Viceré ${ }^{56,57}$ S. Vinciguerra, ${ }^{44}$ D. J. Vine, ${ }^{49}$ J.-Y. Vinet, ${ }^{52}$ S. Vitale, ${ }^{10}$ T. Vo,${ }^{35}$ H. Vocca, ${ }^{32,33}$ C. Vorvick, ${ }^{37}$ D. Voss,${ }^{5}$ W. D. Vousden, ${ }^{44}$ S. P. Vyatchanin, ${ }^{48}$ A. R. Wade,${ }^{20}$ L. E. Wade,${ }^{132}$ M. Wade,${ }^{132}$ M. Walker, ${ }^{2}$ L. Wallace, ${ }^{1}$ S. Walsh, ${ }^{16,8,29}$ G. Wang, ${ }^{12}$ H. Wang, ${ }^{44}$ M. Wang, ${ }^{44}$ X. Wang, ${ }^{70}$ Y. Wang, ${ }^{50}$ R. L. Ward, ${ }^{20}$ J. Warner,${ }^{37}$ M. Was, ${ }^{7}$ B. Weaver, ${ }^{37}$ L.-W. Wei, ${ }^{52}$ M. Weinert, ${ }^{8}$ A. J. Weinstein, ${ }^{1}$ R. Weiss, ${ }^{10}$ T. Welborn, ${ }^{6}$ L. Wen, ${ }^{50}$ P. Weßels, ${ }^{8}$ T. Westphal, ${ }^{8}$ K. Wette, ${ }^{8}$ J. T. Whelan, ${ }^{12,8}$ D. J. White, ${ }^{86}$ B. F. Whiting, ${ }^{5}$ D. Williams, ${ }^{36}$ R. D. Williams, ${ }^{1}$ A. R. Williamson, ${ }^{91}$ J. L. Willis, ${ }^{133}$ B. Willke, ${ }^{17,8}$ M. H. Wimmer, ${ }^{8,17}$ W. Winkler, ${ }^{8}$ C. C. Wipf, ${ }^{1}$ H. Wittel, ${ }^{8,17}$ G. Woan, ${ }^{36}$ J. Worden, ${ }^{37}$ J. L. Wright, ${ }^{36}$ G. Wu, ${ }^{6}$ J. Yablon, ${ }^{82}$ W. Yam, ${ }^{10}$ H. Yamamoto, ${ }^{1}$ C. C. Yancey, ${ }^{62}$ M. J. Yap, ${ }^{20}$ H. Yu, ${ }^{10}$ M. Yvert ${ }^{7}$ A. Zadrożny, ${ }^{110}$ L. Zangrando, ${ }^{42}$ M. Zanolin, ${ }^{97}$ J.-P. Zendri, ${ }^{42}$ M. Zevin, ${ }^{82}$ F. Zhang, ${ }^{10}$ L. Zhang, ${ }^{1}$ M. Zhang, ${ }^{119}$ Y. Zhang, ${ }^{112}$ C. Zhao, ${ }^{50}$ M. Zhou, ${ }^{82}$ Z. Zhou, ${ }^{82}$ X. J. Zhu, ${ }^{50}$ M. E. Zucker, ${ }^{1,10}$ S. E. Zuraw, ${ }^{101}$ and J. Zweizig ${ }^{1}$

(LIGO Scientific Collaboration and Virgo Collaboration)

${ }^{1}$ LIGO, California Institute of Technology, Pasadena, California 91125, USA

${ }^{2}$ Louisiana State University, Baton Rouge, Louisiana 70803, USA

${ }^{3}$ Università di Salerno, Fisciano, I-84084 Salerno, Italy

${ }^{4}$ INFN, Sezione di Napoli, Complesso Universitario di Monte S. Angelo, I-80126 Napoli, Italy

${ }^{5}$ University of Florida, Gainesville, Florida 32611, USA

${ }^{6}$ LIGO Livingston Observatory, Livingston, Louisiana 70754, USA

${ }^{7}$ Laboratoire d'Annecy-le-Vieux de Physique des Particules (LAPP), Université Savoie Mont Blanc, CNRS/IN2P3, F-74941 Annecy-le-Vieux, France

${ }^{8}$ Albert-Einstein-Institut, Max-Planck-Institut für Gravitationsphysik, D-30167 Hannover, Germany

${ }^{9}$ Nikhef, Science Park, 1098 XG Amsterdam, Netherlands

${ }^{10}$ LIGO, Massachusetts Institute of Technology, Cambridge, Massachusetts 02139, USA

${ }^{11}$ Instituto Nacional de Pesquisas Espaciais, 12227-010 São José dos Campos, São Paulo, Brazil

${ }^{12}$ INFN, Gran Sasso Science Institute, I-67100 L'Aquila, Italy

${ }^{13}$ INFN, Sezione di Roma Tor Vergata, I-00133 Roma, Italy

${ }^{14}$ Inter-University Centre for Astronomy and Astrophysics, Pune 411007, India

${ }^{15}$ International Centre for Theoretical Sciences, Tata Institute of Fundamental Research, Bangalore 560012, India

${ }^{16}$ University of Wisconsin-Milwaukee, Milwaukee, Wisconsin 53201, USA

${ }^{17}$ Leibniz Universität Hannover, D-30167 Hannover, Germany

${ }^{18}$ Università di Pisa, I-56127 Pisa, Italy

${ }^{19}$ INFN, Sezione di Pisa, I-56127 Pisa, Italy

${ }^{20}$ Australian National University, Canberra, Australian Capital Territory 0200, Australia

${ }^{21}$ The University of Mississippi, University, Mississippi 38677, USA

${ }^{22}$ California State University Fullerton, Fullerton, California 92831, USA

${ }^{23}$ LAL, Université Paris-Sud, CNRS/IN2P3, Université Paris-Saclay, 91400 Orsay, France

${ }^{24}$ Chennai Mathematical Institute, Chennai 603103, India

${ }^{25}$ Università di Roma Tor Vergata, I-00133 Roma, Italy

${ }^{26}$ University of Southampton, Southampton SO17 1BJ, United Kingdom

${ }^{27}$ Universität Hamburg, D-22761 Hamburg, Germany

${ }^{28}$ INFN, Sezione di Roma, I-00185 Roma, Italy

${ }^{29}$ Albert-Einstein-Institut, Max-Planck-Institut für Gravitationsphysik, D-14476 Potsdam-Golm, Germany

${ }^{30}$ APC, AstroParticule et Cosmologie, Université Paris Diderot, CNRS/IN2P3, CEA/Irfu,

Observatoire de Paris, Sorbonne Paris Cité, F-75205 Paris Cedex 13, France 
${ }^{31}$ Montana State University, Bozeman, Montana 59717, USA

${ }^{32}$ Università di Perugia, I-06123 Perugia, Italy

${ }^{33}$ INFN, Sezione di Perugia, I-06123 Perugia, Italy

${ }^{34}$ European Gravitational Observatory (EGO), I-56021 Cascina, Pisa, Italy

${ }^{35}$ Syracuse University, Syracuse, New York 13244, USA

${ }^{36}$ SUPA, University of Glasgow, Glasgow G12 8QQ, United Kingdom

${ }^{37}$ LIGO Hanford Observatory, Richland, Washington 99352, USA

${ }^{38}$ Wigner RCP, RMKI, H-1121 Budapest, Konkoly Thege Miklós út 29-33, Hungary

${ }^{39}$ Columbia University, New York, New York 10027, USA

${ }^{40}$ Stanford University, Stanford, California 94305, USA

${ }^{41}$ Università di Padova, Dipartimento di Fisica e Astronomia, I-35131 Padova, Italy

${ }^{42}$ INFN, Sezione di Padova, I-35131 Padova, Italy

${ }^{43}$ CAMK-PAN, 00-716 Warsaw, Poland

${ }^{44}$ University of Birmingham, Birmingham B15 2TT, United Kingdom

${ }^{45}$ Università degli Studi di Genova, I-16146 Genova, Italy

${ }^{46}$ INFN, Sezione di Genova, I-16146 Genova, Italy

${ }^{47}$ RRCAT, Indore, MP 452013, India

${ }^{48}$ Faculty of Physics, Lomonosov Moscow State University, Moscow 119991, Russia

${ }^{49}$ SUPA, University of the West of Scotland, Paisley PA1 2BE, United Kingdom

${ }^{50}$ University of Western Australia, Crawley, Western Australia 6009, Australia

${ }^{51}$ Department of Astrophysics/IMAPP, Radboud University Nijmegen, 6500 GL Nijmegen, Netherlands

${ }^{52}$ Artemis, Université Côte d'Azur, CNRS, Observatoire Côte d'Azur, CS 34229 Nice cedex 4, France

${ }^{53}$ MTA Eötvös University, “Lendulet” Astrophysics Research Group, Budapest 1117, Hungary

${ }^{54}$ Institut de Physique de Rennes, CNRS, Université de Rennes 1, F-35042 Rennes, France

${ }^{55}$ Washington State University, Pullman, Washington 99164, USA

${ }^{56}$ Università degli Studi di Urbino "Carlo Bo," I-61029 Urbino, Italy

${ }^{57}$ INFN, Sezione di Firenze, I-50019 Sesto Fiorentino, Firenze, Italy

${ }^{58}$ University of Oregon, Eugene, Oregon 97403, USA

${ }^{59}$ Laboratoire Kastler Brossel, UPMC-Sorbonne Universités, CNRS, ENS-PSL Research University, Collège de France, F-75005 Paris, France

${ }^{60}$ Astronomical Observatory Warsaw University, 00-478 Warsaw, Poland

${ }^{61}$ VU University Amsterdam, 1081 HV Amsterdam, Netherlands

${ }^{62}$ University of Maryland, College Park, Maryland 20742, USA

${ }^{63}$ Center for Relativistic Astrophysics and School of Physics, Georgia Institute of Technology, Atlanta, Georgia 30332, USA

${ }^{64}$ Institut Lumière Matière, Université de Lyon, Université Claude Bernard Lyon 1, UMR CNRS 5306, 69622 Villeurbanne, France

${ }^{65}$ Laboratoire des Matériaux Avancés (LMA), IN2P3/CNRS, Université de Lyon, F-69622 Villeurbanne, Lyon, France

${ }^{66}$ Universitat de les Illes Balears, IAC3-IEEC, E-07122 Palma de Mallorca, Spain

${ }^{67}$ Università di Napoli "Federico II," Complesso Universitario di Monte S. Angelo, I-80126 Napoli, Italy

${ }^{68}$ NASA/Goddard Space Flight Center, Greenbelt, Maryland 20771, USA

${ }^{69}$ Canadian Institute for Theoretical Astrophysics, University of Toronto, Toronto, Ontario M5S 3H8, Canada

${ }^{70}$ Tsinghua University, Beijing 100084, China

${ }^{71}$ Texas Tech University, Lubbock, Texas 79409, USA

${ }^{72}$ The Pennsylvania State University, University Park, Pennsylvania 16802, USA

${ }^{73}$ National Tsing Hua University, Hsinchu City, 30013 Taiwan, Republic of China

${ }^{74}$ Charles Sturt University, Wagga Wagga, New South Wales 2678, Australia

${ }^{75}$ University of Chicago, Chicago, Illinois 60637, USA

${ }^{76}$ Caltech CaRT, Pasadena, California 91125, USA

${ }^{77}$ Korea Institute of Science and Technology Information, Daejeon 305-806, Korea

${ }^{78}$ Carleton College, Northfield, Minnesota 55057, USA

${ }^{79}$ Università di Roma “La Sapienza," I-00185 Roma, Italy

${ }^{80}$ University of Brussels, Brussels 1050, Belgium

${ }^{81}$ Sonoma State University, Rohnert Park, California 94928, USA 


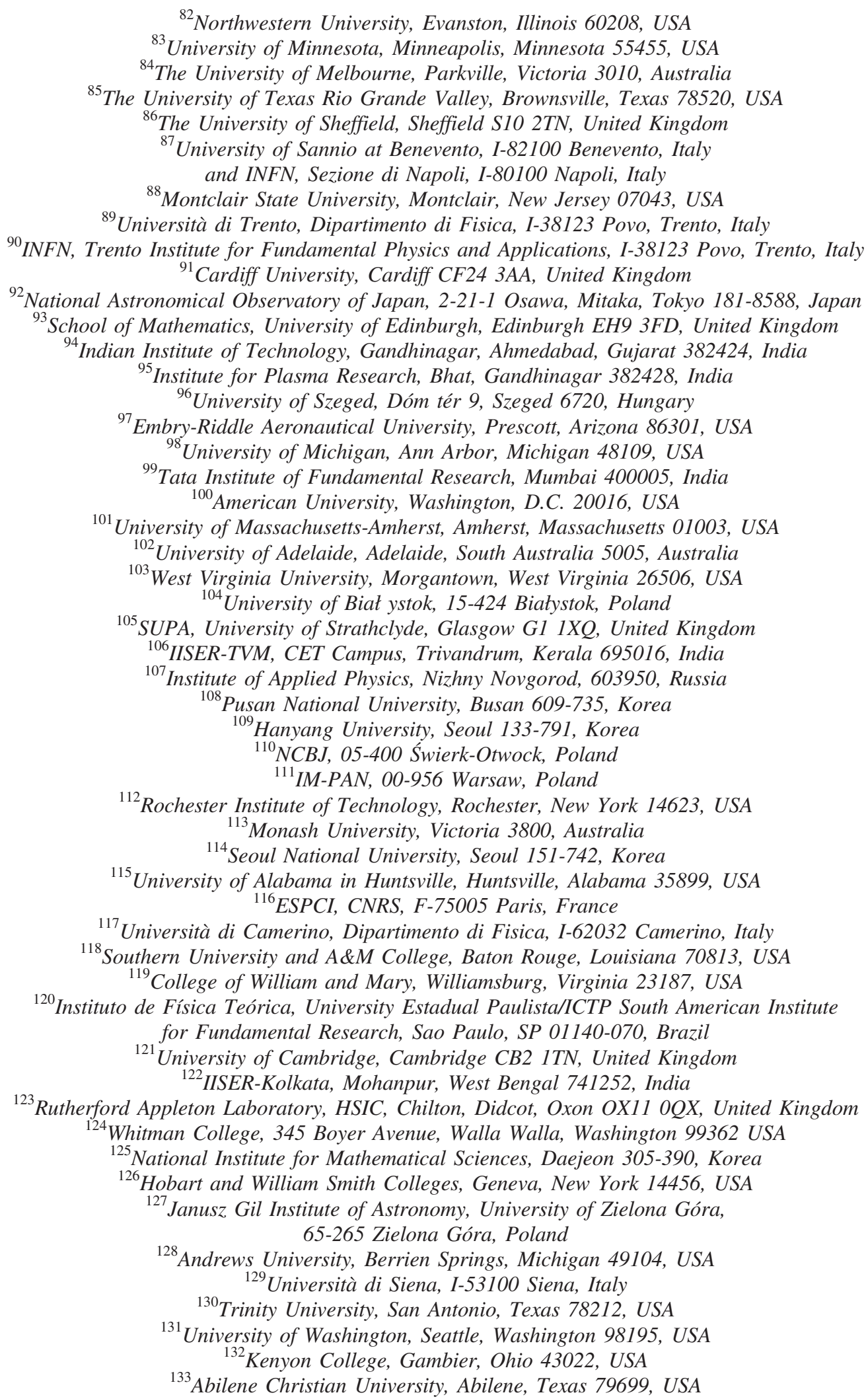

${ }^{\dagger}$ Deceased, May 2015.

${ }^{\ddagger}$ Deceased, March 2015. 